March 8, 1989

\title{
ELECTROWEAK SYMMETRY BREAKING STUDIES AT THE PP COLLIDERS OF THE 1990'S AND BEYOND
} \author{
June 10-27, 1988 \\ CCSEM, Erice-Trapani, Sicily \\ To be published in the proceedings. \\ Michael S. Chanowitz \\ Theoretical Physics Group \\ Physics Division \\ Laurence Berkeley Laboratory \\ University of California \\ 1 Cyclotron Road \\ Berkeley, CA 94720
}

Two lectures presented at the INFN ELOISATRON Workshop

\begin{abstract}
Within the conventional framework of a spontaneously broken gauge theory, general principles establish that the electroweak symmetry is broken by a new force that may be weak with associated new quanta below $1 \mathrm{TeV}$ or strong with quanta above $1 \mathrm{TeV}$. The SSC parameters, $\sqrt{s}=40 \mathrm{TeV}$ and $\mathcal{C}=10^{33} \mathrm{~cm}^{-2} \mathrm{~s}^{-1}$, define a minimal facility with assured capability to observe the signals of symmetry breaking by a strong force above $1 \mathrm{TeV}$. Foreseeable luminosity upgrades would not be able to compensate a much lower collider energy for these physics signals. If the strong $W W$ scattering signal were seen at the SSC in the 1990's it would provide a clear imperative for a collider with the physics reach of the ELOISATRON to begin detailed studies of the new force and quanta early in the next century.
\end{abstract}

- This work wa supported by the Director, Office of Energy Research, Office of High Energy and Nuclear Physica, Division of High Energy Fhysics of the U.S. Department of Energy under Contract DE-AC0376SF00098. 


\section{SYMMETRY BREAKING AND THE TEV SCALE}

In the energy landscape reaching up to the Planck scale, one landmark stands out clearly at the TeV scale. General arguments tell us that the origin of $W$ and $Z$ masses, the mechanism of electroweak symmetry breaking, waits to be discovered at the $\mathrm{TeV}$ scale or below. It is therefore very exciting that we may soon be able to explore this region with multi- $\mathrm{TeV}$ pp collisions.

One purpose of this talk is to review the reasons that a pp collider with the design energy and lurninosity of the $\mathrm{SSC}, \sqrt{3}=40 \mathrm{TeV}$ and $\mathcal{L}=10^{33} \mathrm{~cm}^{-2} \mathrm{~s}^{-1}$, is a minimal facility with an assured capability to point the way to the symmetry breaking mechanism. In particular, such a pp collider will teach us whether electroweak symmetry breaking is due to new, weakly interacting quanta below $1 \mathrm{TeV}$ or to new, strongly interacting quanta above $1 \mathrm{TeV}$. In the latter case the signals at the SSC are observable but small, and an even higher energy facility would be needed to study the new phenomena in detail. This might be a $200 \mathrm{TeV}$ pp collider like the ELOISATRON that is the subject of this workshop and/or a multi $\mathrm{TeV} e^{+} e^{-}$collider of energy $10 \mathrm{TeV}$ or more. Though it may seem reckless to imagine such incredible devices when 16 and $40 \mathrm{TeV}$ pp collider= are still just gleams in their designers' eyes, it is nevertheless clear that symmetry breaking physics at or above $1 \mathrm{TeV}$ would impel us irresistably in this direction.

In this talk I will briefly review the general analysis which identifies the weak boson pair channel $(W W, W Z, Z Z)$ between 1 and $2 \mathrm{TeV}$ as the critical physics signal that we must be able to detect. If we are able to detect this signal, then we will learn from its prs ence or its absence. If present, we learn that symmetry breaking is due to new strongly interacting physics at or above $1 \mathrm{TeV}$, and we will probably see the first signs of the new quanta in the 1-2 TeV signal. If absent, we learn that symmetry breaking is due to weakly interacting quanta below $1 \mathrm{TeV}$, and we will be strongly motivated to redouble the search below $1 \mathrm{TeV}$. itherefore we win whether the signal occurs or not, in contrast to the more typical situation in which a negative result leaves all possibilities open.

The unified theory of weak and electromagnetic interactions ${ }^{1}$ implies the existence of a new (fifth) force and a world of new particles, the "symmetry breaking sector". This force induces spontaneous breaking of the electroweak symmetry, so that the $W$ and $Z$ become massive while the photon remains massless. At the same time the $W$ and $Z$ acquire longitudinal polarization states, which are essentiaily particles (Goldstone bosons) from the symmetry breaking sector. In this sense, three new particles from the symmetry breaking sector have already been discovered: the longitudinal modes $W_{L}^{ \pm}$and $Z_{L}$. They are the pions of this new world of particles.

By measuring $W_{L}$ and $Z_{L}$ scattering amplitudes we therefore measure the strength of the symmetry breaking force. This is done at colliders by means of the process shown in figure (1). The W's are initially virtual (off-shell) and must rescatter to become real (onshell). The number of $W_{L}$ and $Z_{L}$ pairs produced by this mechanism thus measures the 
strength of the unknown force. ${ }^{2}$

Model-independent arguments, based only on symmetry and unitarity, ${ }^{2,3}$ specify the two possibilities: a weak force with associated particles below $1 \mathrm{TeV}$ (Higgs bosons) or a strong force with a rich spectrum of new particles (probably not simply Higgs bosons) above $1 \mathrm{TeV}$. The latter case could be a confining gauge theory, as in technicolor, ${ }^{4}$ or it could be something completely unanticipated.

Deciding between these alternatives is one of the fundamental problems of particle physics, and one which we have the best chance of soiving in the immediate future. The first stage in a systematic approach would be to build a colir:i, r capable of detecting the ${ } V_{L}$ pairs that would arise from figure (1) if the new forcs is strorg i.e., strong $W W$ scattering. Such a device could tell us definitively whether the new physics lies below or above $1 \mathrm{TeV}$. If there is strong $W W$ scattering the lightest resonances would probably appear at $\sim 2 \pm 1$ $\mathrm{TeV}$, i.e., at the onset of the strong scattering domain. The first stage collider might be able to detect the lightest resonances but it would not be capable of detailed studies of the spectrum that would exist above I TeV. It would be as if we knew hadrons existed but had not yet built the Bevatron or the PS to study them. A new higher energy facility would be imperative.

The strong $W W$ scattering signal would, if it exists, emerge between 1 and $2 \mathrm{TeV}$ in the $W W$ invariant mass. Observation of this signal places great demands on the collider luminosity and, especially, on the collider energy. There are two reasons for this extreme sensitivity to the energy: one has to do simply with the size of the signal and the other has to do with the effect of the background on the significance of the signal.

Like the analogous photon-photon scattering process at $e^{+} e^{-}$colliders, WW fusion ${ }^{5}$ places great demands on beam energy since the final state fermions "want" to keep a large fraction of their initial energy. The analogue of the Weiszacker-Williams luminosity function for longitudinal $W$ 's is ${ }^{6,2}$

$$
\frac{\partial \mathcal{L}}{\partial x}=\frac{\alpha^{2}}{16 \pi \sin ^{4} \theta_{w}} \frac{1}{x}\left[(1+x) \ln \frac{1}{x}+2 x-2\right]
$$

where $x=s_{w w} / s_{q q}$. Here $\partial \mathcal{L} / \partial x$ is the effective distribution function to find incident $W_{L}$ beams in colliding quark (or antiquark) beams. The steep dependence on energy is clear in table 1. We see that for fixed sww, doubling the initial quark energy from $\sqrt{s_{q q}}=2 \sqrt{s_{w} w}$ to $4 \sqrt{s W W}$ causes the luminosity to rise by a factor of 20 . The consequences for the $W W$ fusion event rate as a function of collider energy are evident in the results presented in Section 4.

The second aspect of the importance of collider energy has to do with the signal:background ratio. For $\sqrt{s_{w w}} \cong 1-2 \mathrm{TeV}$, the quark distribution functions at LHC and SSC energies tend to force us toward the threshold region in eq. (1.1). In the case of the $W W$ fusion signal we are dealing with a four body final state, $q q \rightarrow q q W W$, so the dependence of the phase space and the cross section is a very steep function of energy. On the other hand the 
dominant backgrounds such as $\bar{q} q \rightarrow W W$ have two body final states and therefore depend much less sensitively on energy. Consequently as we raise the collider energy not only the signal increases but also the signal:background ratio. This means, as discussed more extensively in Section 4, that to gain statistical parity a lower energy collider must be upgraded in luminosity by a ratio that is larger than just the ratio of signal cross sections. In evaluating the trade-off between energy and luminosity, it is also important to keep in mind that the largest gauge boson pair decay modes which are observable at $\mathcal{L}=10^{33} \mathrm{~cm}^{-2} \mathrm{~s}^{-1}$ are probably not observable in the environment of $\mathcal{L} \gg 10^{33}$ (see ref. (7) and Section 4). The task of preparing for physics at $\mathcal{L}=10^{33}$ is already a major challenge.

Unlike other physics goals of multi-TeV colliders which may or may not exist in nature, we know quite generally that the effects of the symmetry breaking sector must emerge in the $W_{L} W_{L}, W_{L} Z_{L}$, and $Z_{L} Z_{L}$ channels at of below $\sim 2 \mathrm{TeV}$ - provided only that we accept the conventional framework of a spontaneously broken gauge theory. The basic physical point is that $W_{L}$ and $Z_{L}$ are essentially (as made explicit by the equivalence theorem discussed in Section 2) the "pions" of the symmetry breaking sector, and like the pions of hairon physics they obey low energy theorems characteristic of the scattering of Goldstone bosons. ${ }^{2}$ If there are no other light (compared to $1 \mathrm{TeV}$ ) particles in the symmetry breaking sector, the low energy scattering amplitudes depend only on the known parameters $G_{F}$ and $\rho=\left(M_{W} / M_{Z} \cos \theta_{W}\right)^{2}$ and not at all on the unknown physics of the symmetry breaking sector, ${ }^{3}$ denoted by its lagrangian $\mathcal{L}_{S B}$. For example, one of the low energy amplitudes is

$$
M\left(W_{L}^{+} W_{L}^{-} \rightarrow Z_{L} Z_{L}\right)=\sqrt{2} G_{F} \frac{s}{\rho} .
$$

Substituting

$$
\sqrt{2} G_{F}=\frac{1}{v^{2}}
$$

where $v=246 \mathrm{GeV}$ is the vacuum expectation value of the standard Higgs boson model ${ }^{1}$ and using the experimental fact that $\rho \cong 1$, eq. (1.2) can be rewritten as

$$
\mathcal{M}\left(W_{L}^{+} W_{L}^{-} \rightarrow Z_{L} Z_{L}\right)=\frac{s}{v^{2}} .
$$

Equation (1.4) has a more than coincidental resemblance to the pion low energy theorem. ${ }^{8}$

$$
\mathcal{U}\left(\pi^{+} \pi^{-} \rightarrow \pi^{0} \pi^{0}\right)=\frac{s}{F_{\pi}^{2}} .
$$

However, as shown in Section 2, eqs. (1.2) and (1.4) are more properly called "intermediate energy theorems" and are only relevant if there is a strongly coupled symmetry breaking sector above $1 \mathrm{TeV}$.

A priori we know neither the mass scale $M_{S B}$ nor the coupling strength $\lambda_{S B}$ of the symmetry breaking sector lagrangian $\mathcal{L}_{S B}$. The low energy theorem provides the correlation 
between the mass scale $M_{S B}$ and the interaction strength $\lambda_{S B}$. Unitarity requires that the amplitude cannot be proportional to $s$ for arbitrarily large $s$, and the most likely scenario (Section 3 ) is that the growth in $s$ is cut off at the mass scale $\|_{S B}$. This observation allows us to identify the strong coupling regime with the mass domain $M_{S B} \gtrsim 1 \mathrm{TeV}$ (see Section 3).

The low energy theorems are the key to a general "no-lose" strategy because they provide an estimate of the scale of $\sigma\left(W_{L} W_{L}\right)$ if $\mathcal{L}_{S B}$ is strongly coupled (I cannot bring myself to use the more accurate term "intermediate energy theorems"). A collider at which we can detect the strong $W W W$ scattering signal is sure to lead us to the symmetry breaking physics, whether strong WIV scattering occurs or not.

For the SSC the crucial observation is that strong $W_{L}, Z_{L}$ scattering is observable at the SSC by virtue of increased yields of gauge boson pairs produced with the $W W$ fusion mechanism shown in figure (1). At the design energy and luminosity these extra gauge boson pairs will be observable above the background sources of gauge boson pairs that are present whether $\mathcal{L}_{S B}$ is a strong or weak coupling theory. The conclusion is the

No-Lose Corollary:

Either there are light $\left(\$ 1 \mathrm{TeV}\right.$ ) particles from $\mathcal{L}_{S B}$ that can be produced and studied directly

and/or

Excess $W W, W Z, Z Z$ production is observable, signaling strongly-coupled $\mathcal{L}_{S B}$ with $M_{S B} Z 1 \mathrm{TeV}$.

For the strong coupling case, if (as in hadron physics) resonances occur when the partial wave amplitudes are $O^{\prime}(1)$, then probably $M_{S B} \leqslant O(2) \mathrm{TeV}$ and the low-lying spectrum of $\mathcal{L}_{S B}$ is (just) visible. However, the 1-2 TeV strong coupling signal would be observable even if $. U_{S B}>>2 \mathrm{TeV}$ and the new particles were too heavy to produce. With $\sqrt{s}=40 \mathrm{TeV}$ and $\mathcal{L}=10^{33} \mathrm{~cm}^{-2} \mathrm{~s}^{-1}$ the SSC would be a minimal pp collider at which the no-lose strategy could be pursued (see Section 4).

In the weak coupling, sub-TeV case there is one known exception that should be menvioned: if $\mathcal{L}_{S B}$ is given by the minimal Higgs model and if the mass happens to lie in the interval $2 m_{t}<m_{H}<2 . M_{W}$, then although $10^{6}$ Higgs would be produced in an SSC year, it is not now known how to detect them in their $H \rightarrow \bar{t} t$ decay mode. ${ }^{9}$ For now the only known way of discovering the Higgs boson in this mass range is to build a $\sqrt{s}>330 \mathrm{GeV} e^{+} e^{-}$ collider with a luminosity of $210^{32} \mathrm{~cm}^{-2} \mathrm{~s}^{-1}$. Even in this rather unlikely scenario (becoming more unlikely as the lower bound on $m_{t}$ rises toward $M_{W}$ ), the SSC would contribute to our understanding of symmetry breaking by verifying the absence of the excess gauge boson pairs 
associated with new strong interactions. (Strong coupling models might have light scalars that would approximately mimic a light Higgs boson.) In general the absence of additional gauge boson pairs from $W W$ fusion would be our cue for a redoubled search of the sub- $\mathrm{TeV}$ mass scale.

I want to make a few comments to put the above statements in perspective. TeV scale symmetry breaking physics puts the greatest demands on energy and luminosity. Some of the other physics that has been contemplated is less demanding and less sensitive to the difference between 15 and $40 \mathrm{TeV}$. However, symmetry breaking physics has a special claim on our attention, since while other possible new physics (e.g. compositeness, $Z^{\prime}, \cdots$ ) may or may not ocrur in nature, we know for sure that the electroweak symmetry is broken. The no-lose corculary says that a facility able to "see" the 1-2 TeV signal of strongly-coupled symmetry breaking gives us valuable information about the mass scale $M_{S B}$ of the symmetry breaking physics whether the new particles occur below, within, or above the 1-2 TeV region. As will become clear from the results presented below, a pp collider with $\sqrt{s}=40 \mathrm{TeV}$ and $\mathcal{L}=10^{33} \mathrm{~cm}^{-2} \mathrm{~s}^{-1}$ is just able to observe the $1-2 \mathrm{TeV}$ strong-interaction signal but is not sufficient to study it in detail. In this sense it is an optimal probe, since we would not want to consider a more ambitious facility without first knowing that there is $\mathrm{TeV}-\mathrm{scale}$ physics requiring detailed study. Conversely, and very much to the point of this meeting, if the strong scattering signal is seen it is clear that we will badly need some more ambitious facility.

Though the problem of observing the strong interaction symmetry breaking signal at $\mathrm{TeV} e^{+} e^{-}$colliders has not been as extensively studied, several authors have had a first look at the signal and/or backgrounds. ${ }^{10}$ The conclusion from Bento and Llewellyn-Smith is that the signal of the conservative model discussed below would not be visible at an $e^{+} e^{-}$collider with $\sqrt{s}, \mathcal{L}=2 \mathrm{TeV}, 10^{33} \mathrm{~cm}^{-2} \mathrm{~s}^{-1}$ but requires a collider in the range $\sqrt{\mathrm{s}}, \mathcal{L} \sim$ $3-5 \mathrm{TeV},\left(1-2 \frac{1}{2}\right) 10^{33} \mathrm{~cm}^{-2} \mathrm{~s}^{-1}$

Section 2 reviews relevant aspects of spontanecus symmetry breaking, presents the equivalence theorem which makes precise the connection between the longitudinal modes and the underlying Goldstone bosons, and sketches a proof of the low energy theorems. Section 3 is concerned with the implications of unitarity and a simple strong interaction model of WW scattering based on the low energy theorems. Section 4 surveys the experimental signals for a strongly interacting symmetry breaking sector, considering the LHC, the SSC, and the ELOISATRON. Understanding of the signals and backgrounds is far from complete. Much more work is needed to insure effective utilization of the multi-TeV pp colliders. 


\section{SPONTANEOUS SYMMETRY BREAKING AND LOW ENERGY THEOREMS}

In order to implement spontaneous symmetry breaking, the lagrangian of the symmetry breaking sector, $\mathcal{L}_{S B}$, must possess a global symmetry group $G$ - analogous to the flavor symmetry of QCD - which breaks by asymmetry of the vacuum to a smaller group $H$,

$$
G \rightarrow H
$$

Gauge invariance requires that $G$ include the electroweak $S U(2)_{L} \times U(1)_{Y}$ and that $H$ include the unbroken electromagnetic $U(1)_{E, M}$. For each broken generator of $G$ there is a massless Goldstone boson in the spectrum of $\mathcal{L}_{S B}$. Three of these couple to the weak currents and are denoted $w^{ \pm}, z$. Others, if any, are denoted by $\left\{\phi_{i}\right\}$. Including the electroweak gauge interactions, the Goldstone triplet $w^{ \pm}, z$ become longitudinal gauge boson modes $W_{L}^{ \pm}, Z_{L}$, and the $\left\{\phi_{i}\right\}$ acquire small masses $O\left(g M_{S B}\right)$, becoming "pseudo-Goldstone" bosons.

As an example, for two flavor QCD with massless quarks the global symmetry $G$ is $S U(2)_{L} \times S U(2)_{R}$. After spontaneous symmetry breaking the surviving invariance group is $H=S U(2)_{L+R}$ which is just the isospin group. There are three broken generators, corresponding to the axial generators of $S U(2)_{L-R}$, so that three massless Goldstone bosons emerge, $\pi^{ \pm}$and $\pi^{0}$. If there were no other symmetry breaking physics, $\mathcal{L}_{S B}, \pi^{ \pm}$and $\pi^{0}$ would indeed become longitudinal modes of $W^{ \pm}$and $Z$, which would however have masses of $\sim 30 \mathrm{MeV}$ (see e.g. ref. (10a)).

The statement that the longitudinal modes $W_{L}^{ \pm}, Z_{L}$ are identified with the Goldstone bosons $w^{ \pm}, z$ is given a precise meaning by the equivalence theorem,

$$
M\left(W_{L}\left(p_{1}\right) W_{L}\left(p_{2}\right) \ldots\right)=M\left(w\left(p_{1}\right) w\left(p_{2}\right) \ldots\right)_{R}+O\left(\frac{M_{W}}{E_{i}}\right)
$$

In eq. (2.2) the left side is a gauge-invariant S-matrix element involving longitudinal modes while the right side is the corresponding Goidstone boson amplitude in an $R$ or renormalizeable gauge. As indicated in eq. (2.2) the equivalence holds at energies large compared to the $W$ and $Z$ masses. We can use the equivalence theorem to translate statements about Goldstone boson scattering amplitudes into statements about scattering of longitudinally polarized W's and $Z$ 's.

The equivalence theorem was proved in tree approximation in reference (11) and to all orders in both gauge and symmetry breaking interactions in reference (2) (see also reference (12)). The validity of the theorem to ail orders in $\lambda_{S B}$ is crucial since we wish to apply it when $\mathcal{L}_{S B}$ is strongly interacting and perturbation theory in $\lambda_{S B}$ fails. Intuitively the theorem is a plausible consequence of the Higgs inechanism that transmutes the Goldstone bosons $w$ and $z$ into the longitudinal gauge boson modes $W_{L}$ and $Z_{L}$. This is seen explicitly by the gauge transformation from a renormalizable gauge - in which the Goldstone boson fields appear in the lagrangian - to the unitary gauge in which the Goldstone fields do not appear. Nevertheless the proof to all orders is lengthy and complicated, making use of 
the BRS identities which embody the full content of gauge invariance in nonabelian gauge theories. Here I will only state the theorem and illustrate it with a simple example.

In addition to being useful in the derivation of the $W_{L} W_{L}$ low energy theorems, eq. (2.2) greatly simplifies perturbative calculations for heavy - and therefore strongly coupled Higgs systems (see references $(13,14,15,2))$. For instance, to correctly evaluate heavy Higgs boson production and decay by $W W$ fusion in unitary gauge requires evaluation of many diagrams with "bad" high energy behavior that cancel to give the final result. ${ }^{16-18}$ But to leading order in the strong coupling $\lambda=m_{\mu}^{2} / 2 v^{2}$ it suffices using eq. (2.2) to compute just a few simple diagrams using the interactions of the Higgs scalar potential. The result embodies the cancellations of many diagrams in unitary gauge and trivially has the correct high energy behavior. On the other hand, unitary gauge calculations of Higgs boson production in the s-channel pole approximation ${ }^{19}$ have bad high energy behavior and overestimate the yield for heavy Higgs bosons, $m_{H} \geq 800 \mathrm{GeV}$.

As a simple example, consider the decay of a heavy Higgs boson to $W_{L}^{+} W_{L}^{-}$. In unitary gauge the $H W_{L}^{+} W_{L}^{-}$amplitude is

$$
\mathcal{M}\left(H \rightarrow W_{L}^{+} W_{L}^{-}\right)=g M_{W \epsilon_{L}}\left(p_{1}\right) \cdot \epsilon_{L}\left(p_{2}\right) .
$$

For $m_{H} \gg M_{W}$ we neglect terms of order $M_{W} / m_{H}$, so that $\epsilon_{L}^{\mu}\left(p_{i}\right) \cong p_{i} / M_{W}$ and similarly from $m_{H}^{2}=\left(p_{1}+p_{2}\right)^{2} \cong 2 p_{1} \cdot p_{2}$ we find

$$
\mathcal{M}\left(H \rightarrow W_{L}^{+} W_{L}^{-}\right)=g \frac{m_{H}^{2}}{2 M_{W}}+O\left(\frac{M_{W}}{m_{H}}\right) .
$$

In a renormalizable gauge the corresponding amplitude can be read off (taking care with factors of 2) from the $H w w$ vertex in the Higgs potential

$$
\mathcal{M}\left(H \rightarrow w^{+} w^{-}\right)=2 \lambda v .
$$

Using $M_{W}=\frac{1}{2} g v$ and $m_{H}^{2}=2 \lambda v^{2}$ it is easy to see that eqs. (2.4) and (2.5) are indeed equal up to $M_{W} / m_{H}$ corrections.

The accuracy of the equivalence therrem can be judged in figure (2) taken from Bento and Llewellyn Smith. ${ }^{10}$ The scattering cin ections for $W_{L}^{+} W_{L}^{-} \rightarrow W_{L}^{+} W_{L}^{-}$and $W_{L}^{+} W_{L}^{-} \rightarrow$ $Z_{L} Z_{L}$ are computed in the standard model with $m_{H}=1 \mathrm{TeV}$. The exact calculations are compared with the result obtained from the equivalence theorem. For $\sqrt{s} \approx 800 \mathrm{GeV}$ the agreement is very good and above $1 \mathrm{TeV}$ the two calculations are indistinguishable on the figure.

As an immediate application, consider the case ${ }^{2}$ in which the global symmetry $G$ includes $S U(2)_{L} \times S U(2)_{R}$ and $H$ includes an $S U(2)_{L+R}$. For such theories, which includes the case of the standard Higgs model, $\rho=1$ up to electroweak corrections and we may immediately 
apply the pion low energy theorems that were derived from current algebra for just this case. For pions we have ${ }^{8}$

$$
M\left(\pi^{+} \pi^{-} \rightarrow \pi^{0} \pi^{0}\right)=\frac{s}{F_{\pi}^{2}} \quad s \ll 1 \mathrm{GeV}^{2}
$$

and for $\mathcal{L}_{S B}$ with no particles other than $W, Z$ that are light compared to $M_{S B}$ we would have

$$
M\left(w^{+} w^{-} \rightarrow z z\right)=\frac{s}{v^{2}} \quad s \ll M_{S B}^{2}
$$

where $v=0.246 \mathrm{TeV}$. Using the equivalence theorem this becomes a statement about the scattering of $W_{L}$ and $Z_{L}$ in an intermediate energy domain: ${ }^{2}$

$$
M\left(W_{L}^{+} W_{L}^{-} \rightarrow Z_{L} Z_{L}\right)=\frac{s}{v^{2}} \quad M_{W}^{2} \ll s \ll M_{S B}^{2} .
$$

It is important to stress that the pion low energy theorems were derived before the discovery of QCD. This was possible because they depend only on the symmetry and not on dynamics. Furthermore they are valid to all orders in the strong interactions. The low energy theorems and other current algebra results were important steps toward the discovery of QCD because they provided a reliable method to study the symmetries of a strongly coupled theory that could not be studied using perturbation theory.

The assumptions used above, $G \supset S U(2)_{L} \times S U(2)_{R}$ and $H \supset S U(2)_{L+R}$, are sufficient to guarantee $\rho=1$ to all orders in $\lambda_{S B}$ but they are not necessary conditions for $\rho=1$. We are therefore motivated to derive the low energy theorems for all candidate groups $G$ and $H$ and for all values of $\rho$. The problem we face is equivalent to that of obtaining the pion-pion scattering low energy theorems in the absence of isospin symmetry. The low energy theorems are derived by three different methods: ${ }^{3}$ a perturbative power counting analysis, nonlinear chiral lagrangians, and current algebra. I will sketch the current algebra derivation below. Along with the low energy theorems for general values of $\rho$, the derivation establishes a limited converse to the result quoted above: we find that if $\rho=1$ then the Goldstone boson sertor consisting of $w^{ \pm}, z$ possesses an effective $S U(2)_{L+R}$ symmetry ("custodial" $S U(2)$ ) in the low energy domain $s<M_{S B}^{2}$.

Briefly the derivation is as follows. The global symmetry $G$ must be at least as large as the gauge group, $G \supset S U(2)_{L} \times U(1)_{\gamma}$, so in particular we have the $S U(2)_{L}$ charge algebra

$$
\left[L_{a}, L_{b}\right]=i \varepsilon_{a b c} L_{c}
$$

where the corresponding local currents $L_{a}^{\mu}$ can generally be expanded in terms of the Goldstone triplet $w^{ \pm}, z$ as

$$
L_{a}^{\mu}=\frac{1}{2} r_{a} \varepsilon_{a b c} w_{b} \partial^{\mu} w_{c}-\frac{1}{2} f_{a} \partial^{\mu} w_{a}+\ldots
$$

with terms involving heavy fields omitted. Since $H \supset U(1)_{E M}$ we have $f_{1}=f_{2}$ and $r_{1}=r_{2}$. The $f_{a}$ are analogues of the PCAC constant and determine the gauge boson masses,

$$
M_{w}=\frac{1}{2} g f_{1},
$$




$$
\rho=\left(f_{1} / f_{3}\right)^{2} \text {. }
$$

Corrections are suppressed by inverse powers of order $M_{S B}$ or, because of quantum corrections, by inverse powers of $4 \pi f_{a}$.

It is straightforward to show that the $S U(2)_{L}$ algebra (2.9) requires

$$
\begin{aligned}
& r_{1}=\frac{1}{\sqrt{\rho}}, \\
& r_{3}=2-\frac{1}{\rho},
\end{aligned}
$$

so that the parameters $r_{a}$ and $f_{a}$ in eq. (2.10) are completely determined in terms of $G_{F}$ and $\rho$. In particular, $\rho=1$ implies $f_{1}=f_{2}=f_{3}$ and $r_{1}=r_{2}=r_{3}=1$ which means that the Goldstone boson contributions to $L_{a}^{\mu}$ are the difference of $S U(2)$ vector and axial vector currents. The existence of this vector $S U(2)$ triplet of currents establishes the limited converse alluded to above.

The rest of the derivation is much like the usual current algebra derivation ${ }^{8}$ except that we do not assume an $S U(2)_{L+R}$ isospin invariance. Consequently pole terms which are forbidden by $G$-parity in the pion case are not forbidden here. Assuming that $w^{ \pm}, z$ saturate these pole terms we find Goldstone boson low energy theorems such as

$$
M\left(w^{+} w^{-} \rightarrow z z\right)=\frac{s}{f_{1}^{2}} \frac{1}{\rho} \quad s \ll M_{S B}^{2}
$$

which using (2.11) reduces to (2.7) for the case $\rho=1$. By the equivalence theorem we have then

$$
M\left(W_{L}^{+} W_{L}^{-} \rightarrow Z_{L} Z_{L}\right)=\frac{s}{v^{2}} \frac{1}{\rho} \quad M_{W}^{2} \ll s \ll M_{S B}^{2}
$$

with $v=f_{1} \cong 2 M_{W} / g$. The other two independent amplitudes are

$$
\begin{gathered}
M\left(W_{L}^{+} W_{L}^{-} \rightarrow W_{L}^{+} W_{L}^{-}\right)=-\frac{u}{v^{2}}\left(4-\frac{3}{\rho}\right), \\
M\left(Z_{L} Z_{L} \rightarrow Z_{L} Z_{L}\right)=0,
\end{gathered}
$$

and by crossing we have also

$$
\begin{gathered}
M\left(W_{L}^{ \pm} Z_{L} \rightarrow W_{L}^{ \pm} Z_{L}\right)=\frac{t}{v^{2}} \frac{1}{\rho} \\
M\left(W_{L}^{+} W_{L}^{+} \rightarrow W_{L}^{+} W_{L}^{+}\right)=M\left(W_{L}^{-} W_{L}^{-} \rightarrow W_{L}^{-} W_{L}^{-}\right)=-\frac{s}{v^{2}}\left(4-\frac{3}{\rho}\right) .
\end{gathered}
$$

Like (2.16) eqs. $(2.17-2.20)$ are valid in the intermediate domain $M_{W}^{2} \ll E_{i}^{2} \ll M_{S B}^{2},(4 \pi v)^{2}$. 
It is also instructive to consider the perturbative power counting analysis. ${ }^{3}$ It does not use the equivalence theorem since it is carried out in unitary gauge. This derivation shows directly that the low energy theorems follow from the form of the $S U(2)_{L} \times U(1)_{Y}$ gauge interactions. It reveals an amusing coincidence of the threshold behavior determined by the low energy theorems and the famous "bad" high energy behavior that a massive YangMills theory would have if the masses were not "softly" generated by spontaneous symmetry breaking.

Consider first the minimal $S U(2)_{L} \times U(1)_{Y}$ Higgs model. Though we are ultimately interested in working to order $g^{2}$ in the electroweak gauge coupling and to all orders in the Higgs coupling $\lambda_{S B}$, we begin by examining $W_{L}^{+} W_{L}^{-} \rightarrow Z_{L} Z_{L}$ scattering to tree approximation in both couplings. The tree approximation amplitude in unitary gauge can be decomposed into the sum of a gauge sector term and a symmetry breaking sector term,

$$
\mathcal{M}\left(W_{L}^{+} W_{L}^{-} \rightarrow Z_{L} Z_{L}\right)=\mathcal{M}_{\text {gauge }}+\mathcal{M}_{S B}
$$

We will evaluate the amplitude for $s \gg M_{W}^{2}$. The first term, $\mathcal{M}_{\text {gauge, }}$ is given by the sum of $t$ and $u$ channel $W$ exchanges and by the four point contact interaction. Independent of the nature of the symmetry breaking sector it is a universal function of $M_{W}$ and $\rho$,

$$
M_{\text {gauge }}=\frac{g^{2} s}{4 \rho M_{W}^{2}} \text {. }
$$

The second term, $\mathcal{M}_{S B}$, is in tree approximation just given by s-channel Higgs exchange,

$$
M_{S B}=-\frac{g^{2} s}{4 M_{W}^{2}} \frac{s}{s-m_{H}^{2}} \text {. }
$$

$\mathcal{M}_{S B}$ has the famous "bad" high energy behavior that is cancelled at infinite $s$ by $\mathcal{M}_{S B}$ (since $\rho=1$ in the minimal Higgs model). However for $s \ll m_{f}^{2}, \mathcal{M}_{S B}$ is negligible, so for the low energy dornain $M_{S}^{2} \ll s \ll m_{H}^{2}$ we have

$$
\begin{aligned}
\mathcal{M}\left(W_{L}^{+} W_{L}^{-} \rightarrow Z_{L} Z_{L}\right) & \cong \mathcal{M}_{\text {gauge }} \\
& \cong \frac{g^{2} s}{4 \rho M_{W}^{2}} .
\end{aligned}
$$

Using $M_{W}=g v / 2$ this agrees precisely with eq. (2.16) obtained from the current algebra derivation. So the form of the low energy amplitude is determined by the "bar" ultraviolet behavior of the gauge sector.

Though to this point we have only obtained the theorems in tree approximation for the minimal Higgs model, we can extend the derivation to all orders in $\lambda_{S B}$ for any symmetry breaking sector with no light particles. By power counting one can show that the only strong 
corrections to the low energy amplitudes are absorbed as renormalizations of $M_{W}$ and $\rho$. All other quantum corrections due to the symmetry breaking sector are screened by an extra power of the electroweak coupling constant, $\alpha_{W} / \pi=g^{2} / 4 \pi^{2}$, or they are suppressed by powers of $s / M_{S B}^{2}$, where $M_{S B}$ is the characteristic scale of the spectrum of the symmetry breaking sector. For details see reference (3).

If other light particles are present, such as pseudo-Goldstone bosons, they may or may not cause the low energy theorems to be modified. QCD is an example of a theory in which additional light particles do not cause modifications. That is, the $\pi \pi$ low energy theorems obtained from $S U(2)_{L} \times S U(2)_{R}$ symmetry are not affected by the existence of $K$ and $\eta$ Goldstone bosons if $S U(2)_{L} \times S U(2)_{R}$ is embedded in a spontaneously broken $S U(3)_{L} \times S U(3)_{R}$. 


\section{UNITARITY AND A CONSERVATIVE STRONG INTERACTION MODEL}

Perhaps the most interesting application of the low energy theorems is to use them to estimate the generic signal we should expect if $\mathcal{L}_{S B}$ is a strongly coupled sector at the $\mathrm{TeV}$ scale or above. The problem we face is like the one that physicists of the 1930's would have faced if they knew nothing of nuclei, baryons or other hadrons, but had discovered the pion, measured the PCAC constant $F_{\pi}$, and recognized (!?) the pion as an almost Goldstone boson. They would have then been able to derive the pion-pion low energy theorems, such as eq. (2.6), and the problem would be to use this information to reconstruct the scale of hadron physics. Though it would take a skilled writer of science fiction to make this a plausible plot line for the 1930's, it is precisely the situation we are in today if $\mathcal{L}_{S B}$ is strongly coupled: our pions are the longitudinal modes of $W$ and $Z$, our "PCAC" constant is $v=0.25 \mathrm{TeV}$, and we have the low energy theorems eqs. $(2.16-2.20)$.

The central ingredient in our considerations is unitarity. The linear growth in s, $t, u$ of the amplitudes $(2.16-2.20)$ cannot continue indefinitely or unitarity would be violated. For instance the $W_{L} W_{L} \rightarrow Z_{L} Z_{L}$ amplitude $(2.16)$ is pure s-wave. If we adopt the low energy amplitude (2.16) as a model of the absolute value of the scattering amplitude, then the $J=0$ partial wave amplitude is ${ }^{2}$

$$
\left|a_{0}\left(W_{L}^{+} W_{L}^{-} \rightarrow Z_{L} Z_{L}\right)\right|=\frac{s}{16 \pi v^{2}}
$$

where here and hereafter we set $\rho=1$. Unitarity requires $\left|a_{0}\right| \leq 1$ so we see that the growth of $a_{0}$ must be cut off at a scale $\Lambda$ with

$$
\Lambda \leq 4 \sqrt{\pi} v=1.75 \mathrm{TeV} .
$$

At the cutoff $\sqrt{s}=\Lambda$ the order of magnitude of the amplitude is

$$
\left|a_{0}(\Lambda)\right|=\frac{\Lambda^{2}}{16 \pi v^{2}} .
$$

For $\Lambda \leqslant \frac{1}{2} \mathrm{TeV}$ we have $\left|a_{0}(\Lambda)\right| \ll 1$ indicating weakly interacting symmetry breaking dynamics $\mathcal{L}_{S B}$, while for $\Lambda \gtrsim 1 \mathrm{TeV}$ we have $\left|a_{0}(\Lambda)\right| \cong O(1)$, the hallmark of a strong interaction theory. The most likely dynamics is that the cutoff scale $\Lambda$ is of the order of $M_{S B}$, the typical mass scale of the new quanta. Then for $\Lambda \cong O\left(M_{S B}\right)$ eq. (3.3) generalizes the Higgs model relationship

$$
\frac{\lambda}{8 \pi}=\frac{m_{H}^{2}}{16 \pi v^{2}}
$$

between the mass scale of the new quanta and the strength of the new interactions: weak coupling for $M_{S B} \ll 1 \mathrm{TeV}$ and strong coupling for $M_{S B} \gtrsim O(1) \mathrm{TeV}$.

A weak coupling example is provided by the standard Higgs model with a light Higgs boson, $m_{H} \ll 1 \mathrm{TeV}$, which can be treated perturbatively. Then $a_{0}\left(W_{L} W_{L} \rightarrow Z_{L} Z_{L}\right)$ is 
given in tree approximation by (where I neglect $M_{W}^{2} / s$ )

$$
a_{0}=\frac{-s}{16 \pi v^{2}} \frac{m_{H}^{2}}{s-m_{H}^{2}} \text {. }
$$

For $s \ll m_{H}^{2}$ this agrees with the low energy theorem (3.1) while for $s \gg m_{H}^{2}$ it saturates at the constant value $m_{H}^{2} / 16 \pi v^{2}$. Comparing with (3.3) we see that $m_{H}$ indeed provides the scale for $\Lambda$ in the standard Higgs model.

A strong interaction example is provided by hadron physics. For the $J=I=0$ partial wave, the low energy theorem gives

$$
a_{\infty}\left(\pi^{+} \pi^{-} \rightarrow \pi^{0} \pi^{0}\right)=\frac{s}{16 \pi F_{n}^{2}}
$$

with $F_{\pi}=92 \mathrm{MeV}$. Equation (3.6) would saturate unitarity at $4 \sqrt{\pi} F_{*}=650 \mathrm{MeV}$ which is indeed the order of the hadron mass scale. The $a_{11}$ and $a_{02}$ amplitudes saturate at 1100 and $1600 \mathrm{MeV}$.

The two generic possibilities are illustrated in figure (3). For weak coupling the partial wave amplitudes saturate at values small compared to 1 giving rise to narrow resonances at masses well below $1 \mathrm{TeV}$. For strong coupling they saturate the unitarity limit with broad resonances in the $\mathrm{TeV}$ range.

These results suggest a general experimental strategy to search for the symmetry breaking sector. $W_{L} W_{L}$ fusion at a pp collider probes the interaction of the symmetry breaking sector as shown in figure (1). Since the initial $W_{L} W_{L}$ pair are off mass shell, they must rescatter to appear in the final state as real on-shell particles. Therefore this process measures the strength of the $W_{L} W_{L}$ interaction which we know from the equivalence theorem is essentially the strength of the interaction of the symmetry breaking sector $\mathcal{L}_{S B}$. Counting powers the $W_{L} W_{L}$ fusion amplitude is $O\left(g^{2} \lambda_{S B}\right)$. It must be compared to the $O\left(g^{2}\right)$ background due to $\bar{q} q \rightarrow W W$. The gauge bosons produced in this way are predominantly transversely polarized, but they cannot be efficiently separated from the longitudinal pairs at the necessary statistical level. Therefore the $W_{L} W_{L}$ fusion signal can be visible above the $\bar{q} q \rightarrow W W$ background only if $\lambda_{S B}=O(1)$, that is, if $\mathcal{L}_{S B}$ is strongly interacting.

We will see in detail in Section 4 that for the $Z Z, W Z$, and $W^{+} W^{-}$channels, the signal can only emerge over the $\bar{q} q$ annihilation backgrounds for $M_{w w}>1 \mathrm{TeV}$. This in turn requires a pp collider of at least the SSC design parameters, $\sqrt{s}=40 \mathrm{TeV}$ and $\mathcal{L}=10^{33} \mathrm{~cm}^{-2} \mathrm{~s}^{-1}$. It is unlikely that lower energy can be sufficiently compensated by higher luminosity for this particular physics, as discussed in Section 4.

The strategy then is to look for an excess of gauge boson pair events with $M_{W w}$ between 1 and $2 \mathrm{TeV}$. If there is no excess then we learn that $\mathcal{L}_{S B}$ is weakly interacting and that the new physics, probably Higgs bosons, lies below $1 \mathrm{TeV}$. If an excess is found we learn that $\mathcal{L}_{S B}$ is strongly interacting and that the new quanta lie above $1 \mathrm{TeV}$. 
Since the low energy theorems for pion scattering apply approximately for $\sqrt{s} \lessgtr 300$ $\mathrm{MeV}$, we guess that the $W_{L} W_{L}$ theorems may apply out to $\sqrt{s} \leqslant 300 \mathrm{MeV} \times v / F_{\pi} \cong 1 \mathrm{TeV}$. In the next section we consider the experimental implications of a crude but conservative model ${ }^{2}$ of strong $W_{L} W_{L}$ scattering based on the low energy theorems (2.16-2.20). As in eq. (3.1) we use the low energy theorems as the model for the absolute value of the relevant partial wave amplitudes which are $a_{J I}=a_{00} a_{11}, a_{02}$ where $I$ denotes the effective low energy custodial $S U(2)$. Above the energy at which partial wave unitarity is saturated, the amplitudes are set equal to one. For $a_{00}$ saturation occurs at $\sqrt{16 \pi v^{3}}=1.7 \mathrm{TeV}$, eq. (3.2), so in that case the model is

$$
\left|a_{00}(s)\right|=\frac{s}{16 \pi v^{2}} \theta\left(16 \pi v^{2}-s\right)+1 \cdot \theta\left(s-16 \pi v^{2}\right) .
$$

The detailed form is not critical, the essential point being to extrapolate snoothly from the known behavior at low energy into the domain above $1 \mathrm{TeV}$ where the amplitude becomes strong, of order 1, without assuming enhanced resonant behavior.

The model is conservative in that it assumes no resonant behavior. For instance, the $Z Z$ continuum signal for $M_{Z Z}>1 \mathrm{TeV}$ is only half the corresponding signal from the $1 \mathrm{TeV}$ Higgs boson. Comparatively little is gained at the SSC from the high energy region where $\left|a_{J}\right|=1$ because of the rapidly falling luminosities at the relevant collider energies. The model is also conservative in that only the leading partial waves, $I, J=(0,0),(1,1),(2,0)$, are kept while higher partial waves are set to zero.

Figure (4) from reference (20) shows how well the model fits $\pi \pi$ scattering data. The "dictionary" is that $\sim 650 \mathrm{MeV}$ corresponds to the unitarity limit at $1.75 \mathrm{TeV}$. Our model corresponds to curve $a$ in the plots of $\left|T_{0}^{0}\right|,\left|T_{1}^{1}\right|$, and Re $T_{0}^{2}$ (since $T_{0}^{2}$ is exotic in QCD, the imaginary part is negligible below $1 \mathrm{GeV}$ ). The model describes the trend of the 0,0 channel well. In the 1,1 channel it underestimates the data by a large factor, as a result of the rho meson. In the 2, 0 channel there is considerable disagreement between different experiments; the model interpolates the data out to $\sim 600 \mathrm{MeV}$ beyond which it overestimates the data. At the SSC this is not as serious as it might seem since $\sqrt{s}_{n \pi}>600 \mathrm{MeV}$ corresponds to $\sqrt{s}_{W W}>1.7 \mathrm{TeV}$ where the $W W$ luminosity is small.

In the next section we will consider the experimental signals (and backgrounds) of a strongly interacting symmetry breaking sector for pp collider energies corresponding to the LHIC, the SSC, and the ELOISATRON. 


\section{EXPERIMENTAL SIGNALS FOR STRONG INTERACTION MODELS}

In this section we consider the experimental signals of a strongly interacting symmetry breaking sector. An important general lesson emerges from technicolor models: ${ }^{21}$ the Higgs mechanism does not require the existence of a physical Higgs boson. As shown in Sections 2 and 3 the generic signal of a strongly interacting $\mathcal{L}_{S B}$ is strong $W_{L} W_{L}$ scattering between 1 and $2 \mathrm{TeV}$, giving rise by the mechanism of figure (1) to production of longitudinally polarized gauge boson pairs in all channels: $Z Z, W^{ \pm} Z, W^{+} W^{-}, W^{+} W^{+}, W^{-} W^{-}$. (The longitudinal polariztion of the $W$ 's provides little leverage against the predominantly transversely polarized W's in the $\bar{q} q \rightarrow W W$ background; I do not assume polarization information in the results given here.) For a collider powerful enough to observe this signal we have a No-Lose Corollary: if the signal is not present we learn that the symmetry breaking sector is below $1 \mathrm{TeV}$, probably in the form of one or more Higgs basons.

In this section we will review some of the possible experimental signals. We will see that a collider with the SSC energy and luminosity is a minimal configuration for the 1-2 TeV signals. A pp collider with half the energy would not suffice, nor does it seem practicable for this purpose to compensate the lower energy with higher luminosity. (The necessary luminosity upgrade would be of order 150 to 600 , i. e., $\mathcal{L}=1.5 \cdot 10^{35}$ to $6 \cdot 10^{35} \mathrm{~cm}^{-2} \mathrm{~s}^{-1}$, as discussed below.) The section begins with some general remarks about the relationship of collider energy and luminosity to the "reach" for TeV-scale symmetry breaking signals. Next is a survey of the cross sections and discovery strategies for the pp colliders projected for the 1990's. Finally I present estimates of the cross sections to be expected at a $200 \mathrm{TeV}$ pp collider. The $W W$ fusion mechanism of figure (1) has an important consequence fo: the design of such a pp collider (or for $\gtrsim 10 \mathrm{TeV} e^{+} e^{-}$collider): like the more familiar $\gamma \gamma$ cross section it increases with energy, rather than falling like $1 / s$ as is typical of annihilation and hard scattering processes. As a result, $\mathcal{L}=10^{33} \mathrm{~cm}^{-2} \mathrm{~s}^{-1}$ would suffice at these 21'st century "hypercolliders" to ensure large yields of TeV-scale quanta from a strongly interacting symmetry breaking sector. (Of course higher luminosity will be welcome if 21 'st century experimenters are able to cope with it.)

\subsection{Energy, Luminosity, and Physics Resch}

The four-body phase space of the $W W$ fusion process, $q q \rightarrow q q W W$, increases steeply with energy near threshold, which is in fact the region inhabited by the LHC and SSC for $\sqrt{s_{w W}} \gtrsim 1 \mathrm{TeV}$. The result is that the cross sections reviewed below in Section 4.2 are an order of magnitude larger for the SSC than the LHC. The background process $\bar{q} q \rightarrow W L V$ is two-body and therefore does not rise as steeply with energy in this region. This fact cannot be ignored in appraising the relationship of energy and luminosity.

To what extent can lower energy be compensated by higher luminosity to obtain equivalent reach for TeV symmetry breaking physics? An answer requires consideration of two factors: 
1) what luminosity upgrade would be needed if all the same measurements could be carried out at $\mathcal{L}=10^{33} \mathrm{~cm}^{-2} \mathrm{~s}^{-1}$ and at $\mathcal{L} \gg 10^{33}$ ?

2) what is the factor resulting from decay modes which are detectable at $\mathcal{L}=10^{33}$ but not at much higher luminosity?

We consider these two factors in turn.

Suppose first that it is actually possible to make the same experimental observations at luminosities far above $10^{33} \mathrm{~cm}^{-2} \mathrm{~s}^{-1}$ as at $10^{33}$. Let us first compare two colliders with different energies, $E_{2}>E_{1}$, and the same luminosity, say $\mathcal{L}_{1}=\mathcal{L}_{2}=10^{33} \mathrm{~cm}^{-2} \mathrm{~s}^{-1}$. The physics reach is determined not simply by the number of events in the signal $S_{i}$ but by its statistical significance, proportional to $S_{i} / B_{i}^{1 / 2}$ where $B_{i}$ is the number of background events. The steeper energy dependence of the signals $S_{i}$ than the backgrounds $B_{i}$ implies greater statistical significance at the higher energy collider,

$$
\frac{S_{2}}{B_{2}^{1 / 2}}>\frac{S_{1}}{B_{1}^{1 / 2}}
$$

Next suppose that we upgrade the luminosity of the lower energy collider from $\mathcal{L}_{1}=\mathcal{L}_{2}$ to $\mathcal{L}_{1}^{\prime}$ by a large factor $\mathcal{R}$,

$$
\mathcal{R} \equiv \frac{\mathcal{L}_{1}^{\prime}}{\mathcal{L}_{2}} \gg 1
$$

so that the two machines achieve statistical parity,

$$
\frac{S_{2}}{B_{2}^{1 / 2}}=\frac{S_{1}^{\prime}}{B_{1}^{\prime 1 / 2}}=\frac{\mathcal{R} S_{1}}{\left(\mathcal{R} B_{1}\right)^{1 / 2}}
$$

We see then that the upgrade factor $\mathcal{R}$ must satisfy

$$
\mathcal{R}=\left(\frac{S_{2}}{S_{1}}\right)^{2}\left(\frac{B_{1}}{B_{2}}\right)
$$

Here $S_{i}$ and $B_{i}$ are always the number of signal and background events before upgrade, i.e., for $\mathcal{L}_{1}=\mathcal{L}_{2}$. Therefore the ratios $S_{2} / S_{1}$ and $B_{3} / B_{1}$ are equal to the ratios of the signal and background cross sections at the two energies.

Physics "reach" is by definition the largest mass signal that could be observed with some minimum level of significance, e.g., 3o. For any such signal eq. (4.3) is then the criterion for two colliders to have equal reach and eq. (4.4) gives the luminosity upgrade needed for the lower energy collider. We can use the signal and background cross sections presented below to evaluate the upgrade factor $R$ for specific TeV-scale phenomena. For example, considering 
the $Z Z$ cross sections in tables $2-4$ for the strong scattering model and the $\bar{q} q$ annihilation backgrounds, the upgrade factors are

$$
\left(\frac{S^{2}}{B}\right)_{16 \mathrm{TeV}}:\left(\frac{S^{2}}{B}\right)_{40 \mathrm{TeV}}:\left(\frac{S^{2}}{B}\right)_{200 \mathrm{TeV}} \cong \mathrm{i}: 25: 5 \mathrm{uv}
$$

Assuming the same detection capability, a $16 \mathrm{TeV}$ collider with $\mathcal{L}=2.5 \cdot 10^{34} \mathrm{~cm}^{-2} \mathrm{~s}^{-1}$ would be equivalent to a $40 \mathrm{TeV}$ collider with $\mathcal{L}=10^{33}$. A $40 \mathrm{TeV}$ collider would need $\mathcal{C}=2 \cdot 10^{34}$ to be equivalent to a $200 \mathrm{TeV}$ collider with $\mathcal{L}=10^{33}$.

Under the experimental conditions likely for the 1990's the advantage of $40 \mathrm{TeV}$ over 16 $\mathrm{TeV}$ is much greater than these idealized estimates suggest. In Section 4.2 we consider the particular final states that are likely to be detectable at $\mathcal{L}=10^{33} \mathrm{~cm}^{-2} \mathrm{~s}^{-1}$. These include most certainly the leptonic final states $Z Z \rightarrow \ell^{+} \ell^{-}+\ell^{+} \ell^{-}, Z Z \rightarrow \ell^{+} \ell^{-}+\bar{\nu} \nu, W W \rightarrow \ell \nu+\ell \nu$ (eapecially like-charged) and perhaps also the mixed lepton-hadron final states $W W \rightarrow$ $\ell \nu+\bar{q} q$. Allowing $\ell=e$ or $\mu$ but not $\tau$ and $\bar{q} q=u \bar{d}$ or $c \bar{s}$ but not $t \bar{b}$, the branching ratios for these four decays are respectively $4.4 \cdot 10^{-3}, 2.6 \cdot 10^{-2}, 2.8 \cdot 10^{-2}$, and $1.7 \cdot 10^{-1}$. Because of unavoidable effects of event pile-up it was concluded in the high luminosity option study for the $\mathrm{LHC}^{7}$ that only the first ("gold-plated") decays, $Z Z \rightarrow \ell^{+} \ell^{-}+e^{+} e^{-}$, could be detected for $\mathcal{L} Z 5 \cdot 10^{34}$. Furthermore, as is carefully noted in reference (7), it is not clear that detectors capable of electron identification could survive radiation damage at $\mathcal{L} \geq 5 \cdot 10^{34}$. If not then only $Z Z \rightarrow \mu^{+} \beta^{-}+\mu^{+} \mu^{-}$would be detectable, reducing the branching ratio by another factor four to $1.1 \cdot 10^{-3}$. While one cannot give a precise number without detailed simulations to assess the relative efficiencies of the different decay channels, it is clear from the branching ratios into leptonic final states that a $40 \mathrm{TeV}$ collider gains another order of magnitude advantage from the "silver-plated" decays that include neutrinos in the final state. The advantage would be much larger still if the mixed lepton-hadron decays prove to be detectable. (The latter were also judged to be undetectable at high luminosity in reference (7).)

Assuming electron identification it was concluded in reference 7 that the LHC Higgs boson reach could be raised from $m_{H}=600 \mathrm{GeV}$ at $\mathcal{L}=10^{33}$ using $Z Z \rightarrow \bar{\ell} \ell+\bar{\nu} \nu$ to $m_{H}=800 \mathrm{GeV}$ at $\mathcal{C}=5 \cdot 10^{34}$ with $Z Z \rightarrow \ell^{+} \ell^{-}+\ell^{+} \ell^{-}$assuming $\ell=e$ or $\mu$. Since the $1-2$ $\mathrm{TeV}$ continuum signal of the strong scattering model is much smaller than even the $1 \mathrm{TeV}$ Higgs boson signal, the no-lose criterion is not met even assuming electron identification. (E.g., the strong scattering model for $Z Z$ would then predict 11 signal events in $Z Z \rightarrow$ $e^{+} e^{-} / \mu^{+} \mu^{-}+e^{+} e^{-} / \mu^{+} \mu^{-}$for $10^{7}$ sec. The background would be $\sim 30$ events assuming $\bar{q} q \rightarrow Z Z$ is augmented $30 \%$ by $g g \rightarrow Z Z$.)

Combining the effect of detectability with the $R$ factor, eq. (4.5), the effective luminosity for the strong scattering model signals of a $40 \mathrm{TeV}$ collider is at least two orders of magnitude larger than that of a $16 \mathrm{TeV}$ collider and is nearly three orders of magnitude larger if electrons cannot be identified for $\mathcal{L}=10^{33}$. 


\subsection{Experimental Signals at 16 and $40 \mathrm{TeV}$}

We consider what might actually be observed at the LHC and SSC if $\mathcal{L}_{S B}$ is a strongly interacting theory. The generic strong interaction signal is longitudinally polarized $W, Z$ pairs produced by $W W$ fusion, figure (1). The $W$ polarization does not provide much leverage against the predominantly transverse $\bar{q} q \rightarrow W W$ background, and I will not assume polarization information in the results given here. Since the irreducible background is $\mathcal{M}(\bar{q} q \rightarrow W W)=O\left(g^{2}\right)$ while the signal is $\mathcal{M}(q q \rightarrow q q W W)=O\left(g^{2} \lambda_{S B}\right)$, we expect a discernible signal if and only if $\mathcal{L}_{S B}$ is strongly interacting, $\lambda_{S B}=O(1)$. The signal may occur in $W^{+} W^{-}$and $Z Z$, as for the standard Higgs boson, but also more generally in $W^{ \pm} Z$ and even $W^{+} W^{+}$and $W^{-} W^{-}$( $" I^{n}=1$ and 2 channels) as in eqs. (2.16-2.20).

To compute the expected yields we must convolute the quark luminosity distribution functions with the luminosity distribution to find longitudinally polarized gauge bosons in the incident $q$ 's or $\bar{q}$ 's, convoluted finally with the $2 \rightarrow 2$ scattering cross section of the longitudinally polarized gauge bosons:

$$
\sigma\left(p p \rightarrow Z_{L} Z_{L}+\cdots\right)=\left.\left.\int_{\Gamma} \frac{\partial \mathcal{L}}{\partial \tau}\right|_{q q / p p} \cdot \int_{x} \sum_{V_{L}} \frac{\partial \mathcal{L}}{\partial x}\right|_{V_{L} V_{L} / q q} \cdot \sigma\left(V_{L} V_{L} \rightarrow Z_{L} Z_{L}\right)
$$

where $\tau=s_{q q} / s_{p p}$ and $x=s_{z z} / s_{q q}$. In the effective $W$ approximation the luminosity function for $W_{L}^{+} W_{L}^{-}$pairs is given in eq. (1.1). The $x$ dependence, tabulated in table 1 , shows the strong dependence on the available phase space that will be familiar to practitioners of two photon physics at $e^{+} e^{-}$colliders.

The effective $W$ approximation has been compared with analytical ${ }^{22}$ and numerical evaluations $\mathrm{s}^{23.24,17}$ of Higgs boson production. The most definitive results are probably the analytical calculations of reference (22). They show good agreement for $W W \rightarrow H$ for $m_{H} \geq 500 \mathrm{GeV}$, with errors $\lessgtr O(10 \%)$ and decreasing with increasing $m_{H}$, while for the relatively less important process $Z Z \rightarrow H$ the errors are roughly twice as large.

I will consider three examples of strong interaction symmetry breaking signals: the 1 $\mathrm{TeV}$ Higgs boson, the strong scattering model described in Section 3, and the techni-rho meson resonance expected in technicolor models. Total cross sections (without $Z$ or $W$ decay branching ratios) are shown in figures (5-7) from reference (2). Figures (5) and (6) show the $Z Z$ signals for the strong scattering model of Section 3 and for the $1 \mathrm{TeV}$ Higgs boson respectively, assuming integrated luminosity of $10^{4} \mathrm{pb}^{-1}\left(10^{33} \mathrm{~cm}^{-2} \mathrm{~s}^{-1}\right.$ for $\left.10^{7} \mathrm{~s}\right)$ at $\mathrm{pp}$ colliders with $\sqrt{3}=10,20,30,40 \mathrm{TeV}$. A rapidity cut $|y z|<1.5$ has been imposed in the figures to reduce the $\bar{q} q \rightarrow Z Z$ background which is strongly forward while the signal is relatively isotropic. Figure (7) shows the two signals for the $40 \mathrm{TeV}$ collider as increments to the $\bar{q} q \rightarrow Z Z$ background, again with $|y z|<1.5$. From figure $(i)$ we see the necessity to detect gauge boson pairs at large invariant mass, $m_{v v} \gtrsim O(1) \mathrm{TeV}$, where the signal can emerge from the rapidly falling background.

Yields are presented in tables 2 and 3 for $\sqrt{3}=16$ and $40 \mathrm{TeV}$ with the rapidity cut 
$\left|y_{V}\right|<1.5$ and invariant mass cut $m_{V V}>1.0 \mathrm{TeV}$. The values are taken from reference (2) except that an error in the Higgs boson yield has been corrected (reducing those yields by $\sim 20 \%$ relative to reference $(2))$.

We must include the decay branching ratios to particular final states in order to translate the tables into estimates of the observable yields. The relevant branching ratios for leptonic channels are

$$
\begin{gathered}
B\left(Z Z \rightarrow e^{+} e^{-} / \mu^{+} \mu^{-}+e^{+} e^{-} / \mu^{+} \mu^{-}\right) \cong 0.0044 \\
B\left(Z Z \rightarrow e^{+} e^{--} / \mu^{+} \mu^{-}+\nu \bar{\nu}\right) \cong 0.026 \\
B(W W \rightarrow(e / \mu) \bar{\nu}+(\bar{e} / \bar{\mu}) \nu) \cong 0.028
\end{gathered}
$$

For the "mixed" lepton-hadron channel we have the larger rate

$$
B(W W \rightarrow u \bar{d} / \bar{c}+e \bar{\nu} / \mu \bar{\nu}) \cong 0.17 \text {. }
$$

However the latter has a formidable QCD background from $W+2$ jets, two orders of magnitude larger than the signals even if excellent jet-jet mass resolution is assumed. This background is not shown in the tables.

The cleanest and rarest channel is $H \rightarrow Z Z \rightarrow \ell^{+} \ell^{-} \ell^{+} \ell^{-}$with $\ell=e$ or $\mu$. The background is from $\bar{q} \rightarrow Z Z$ and $g g \rightarrow Z Z$, the latter ${ }^{25}$ proceeding by a quark loop. Recent Monte Carlo simulations suggest that the Higgs boson can be detected in this channel for $m_{H} \leq 300 \mathrm{GeV}$ at the $\mathrm{LHC}^{36}$ and for $m_{H} \leq 600 \mathrm{GeV}$ at the SSC. ${ }^{27}$ (Here and elsewhere unless otherwise stated both $\mathrm{SSC}$ and LHC are assumed to operate at $\mathcal{L}=10^{33} \mathrm{~cm}^{-2} \mathrm{sec}^{-1}$.) At these values of $m_{H}$ the Higgs boson appears as a recognizable peak above the continuum background. For $m_{H}=1 \mathrm{TeV}$ an essentially optimal set of cuts is $y_{z}<1.5$ and $m_{z Z}>1$ $\mathrm{TeV}$, as in the tables. We then obtain 4 signal events over a background (augmented by $50 \%$ for $g g \rightarrow Z Z$ ) of $1 \frac{1}{2}$ events for an integrated luminosity of $10^{4} \mathrm{pb}^{-1}$ at the SSC. (Yields are always quoted per $10^{4} \mathrm{pb}^{-1}$ corresponding to $\mathcal{L}=10^{33} \mathrm{~cm}^{-2} \mathrm{sec}^{-1}$ for $10^{7} \mathrm{sec}$ ) At the LHC the corresponding signal is a factor ten times smaller while the background is about four times smaller. Such a signal at the SSC would not be statistically significant, but augmented by additional years of running and/or results from several experiments it would become significant. It would also be a valuable confirmation of larger signals detected in other channels.

Of course to detect such a structureless signal (for $m_{H}=1 \mathrm{TeV}$ the width is $\Gamma_{H}=$ $0.5 \mathrm{TeV}$ ) it is necessary to know the magnitude of the background, requiring a variety of calibrations in situ to confirm knowledge of the relevant distribution functions and couplings. After such calibration studies are completed the $Z Z$ background should be known to within $30 \%$ uncertainty, ${ }^{27}$ sufficient accuracy given the expected 3:1 signal to background ratio.

Ascending the ladder of rates while descending on the scale of "purity" of signal, we come next to the decay $H \rightarrow Z Z \rightarrow \bar{\ell} \ell \bar{\nu} \nu .^{2,25}$ Monte Carlo simulations have suggested a 
reach in this channel to $m_{H} \lessgtr 600 \mathrm{GeV}$ at the $L \mathrm{LC}^{26}$ and to at least $800 \mathrm{GeV}$ at the $\mathrm{SSC}^{27}$ (A Monte Carlo study for $m_{H}>800 \mathrm{GeV}$ at the SSC has not yet been done). The two studies are not directly comparable because while both considered the $\bar{q} q \rightarrow Z Z$ and $g g \rightarrow Z Z$ backgrounds, only the SSC study considered the background from $Z+$ jet where the jet generates large missing energy, faking a $Z \rightarrow \bar{\nu} \nu$ decay. This background is sharply reduced by cutting on visible hadronic transverse energy on the side opposite to the observed $Z \rightarrow \ell^{+} \ell^{-}$. A very hermetic detector was found to be critical. The analysis may be improved in the future by adding cuts involving event topology and the rapidity distribution of the visible hadronic clusters.

The $1 \mathrm{TeV}$ Higgs boson is probably also observable at the SSC in this mode. Requiring the observed $Z \rightarrow \ell^{+} \ell^{-}$to satisfy $p_{T}>0.45 \mathrm{TeV}$ and $|y|<i .5$, the estimated yield is a signal of 27 events over a ( $\bar{q} q$ or $g g$ ) $\rightarrow Z Z$ background of $\sim 12$ events.

The mixed decay mode, $H \rightarrow W W \rightarrow \bar{\ell} \nu \bar{q} q$ with $\ell=e$ or $\mu$ and $\bar{q} q=u \bar{d}$ or $c \bar{s}$, has a large branching ratio, $2 \times \frac{1}{6} \times \frac{1}{2}=1 / 6$. However the QCD background from $W j j$ is two orders of magnitude bigger than the signal even if we optimistically assume a $5 \%$ measurement of the dijet mass. ${ }^{28}$ Two approaches have been taken to attempt to winnow the signal from this enormous background. One method is to cut on the $p_{T}$ of the jets, using the tendency of the longitudinally polarized $W$ 's from the signal to decay into jets transverse to the $W$ line of flight, unlike the QCD dijets and the transversely polarized $W$ 's that both tend to produce jets along the line of flight. Applying this approach to the SSC for $m_{H}=800 \mathrm{GeV}$ a parton level calculation results in a signal of 500 events over an equal background. ${ }^{29}$ Smaller but still encouraging yields have been reported based on Monte Carlo studies. ${ }^{30}$ The prospects to follow this strategy in the real world are more difficult to assess than for the purely leptonic decays, being more sensitive to detailed aspects of jet physics and detector performance.

The second approach to the mixed modes borrows a trick from photon-photon scattering experiments at $e^{+} e^{-}$colliders where detecting an $e^{ \pm}$near the forward direction is a powerful way to isolate a clean sample of two pioton events. The analogous idea ${ }^{19}$ is to tag the forward jets that occur in $W W$ fusion, $q q \rightarrow q q W W$, with transverse momentum of order $M_{W}$. Of course tagging suffers its own QCD background, due to processes with a real $W \rightarrow \ell \bar{\nu}$ plus a dijet to fake the second $W \rightarrow \bar{q} q$ and one or two jets near the forward direction that fake the tagged quark or quarks. The necessary background colculation has not yet been performed. A recent calculation assuming $100 \%$ efficient tagging for a $700 \mathrm{GeV}$ Higgs boson resulted in 20 events for an LHC year and 160 events for an SSC year ${ }^{31}$ (a "year" is always $10^{7}$ seconds). The QCD backgrounds after tagging were estimated at 12 and 140 events respectively, however the authors remark that their background estimates will probably prove to be small by a factor of 2 . Highly segminted forward calorimeters would be essential.

An additional serious background would occur if $m_{t}>M I_{W}$ so that the top quark decays by $t \rightarrow W b .^{32}$ For $100 \mathrm{GeV} \leq m_{t} \leq 200 \mathrm{GeV}$ the contribution to the $W^{+} W^{-}$continuum from 
$\bar{t} t \rightarrow W^{+} W^{-} \bar{b} b$ would be two orders of magnitude larger than $\bar{q} q \rightarrow W^{+} W^{-}$, eliminating any hope of detecting $H \rightarrow W^{+} W^{-}$. The leptonic decay signals from $H \rightarrow Z Z$ would not be affected.

The experimental signals for the strong scattering model described in Section 3 have not yet been studied at the level of Monte Carlo simulations. Since the continuum signal is shifted to larger values of $M_{Z Z}$ relative to the $1 \mathrm{TeV}$ Higgs boson, il puts even greater stress on the beam energy. The most promising leptonic signal for the $Z Z$ channel is $Z Z \rightarrow \ell^{+} \ell^{-} \bar{\nu} \nu$ with $\ell=e$ or $\mu$. Requiring the observed $Z$ to satisfy $|y|<1.5$ and $p_{T}>0.45 \mathrm{TeV}$, the yield at $\sqrt{s}=40 \mathrm{TeV}$ is 15 events over a background of $\sim 12$ including $g g \rightarrow Z Z$. The corresponding signal at $\sqrt{\mathrm{s}}=16 \mathrm{TeV}$ is 1 event over a background of 4 or 5 . The possibie backiground from $Z+$ jet has not yet been examined but is probably less pernicious than for the $800 \mathrm{GeV}$ Higgs boson considered in reference (27).

Unlike the standard Higgs boson signal, strong interaction continuum signals also occur in the $W^{ \pm} Z$ and like-charge $W W$ channels. $W Z$ can be detected in clean leptonic decays $W Z \rightarrow \ell \nu \ell \bar{\ell}, \ell=e$ or $\mu$, with a $\sim 1 \%$ branching ratio, augmented to $\sim 1.5 \%$ if $W \rightarrow \tau \nu$ is also feasible. Assuming only $e$ 's and $\mu$ 's the expected signal is $\sim 7-1 / 2$ events over a $\bar{q} q \rightarrow W Z$ background of $\sim 3$ after cuts of $|y w, z|<1.5$ and $M_{w z}>1 \mathrm{TeV}$. As discussed below, more significant $W Z$ signals would occur if resonances are present.

The like charge $W^{+} W^{+}+W^{-} W^{-}$signal ${ }^{2,33}$ has no $\bar{q} q$ or $g g$ annihilation backgrounds but does have a background comparable to the signal from e.g. $u u \rightarrow d d W^{+} W^{+}$via single gluon exchange. ${ }^{34}$ The charge can only be detected in leptonic decays so that $M_{W W}$ cannot be measured. An estimate of the strong scattering model signal (defined by $y_{\ell}<3$ and $p_{T}(\ell)>50 \mathrm{GeV}$ ) yielded ${ }^{33} \sim 40$ events at $\sqrt{s}=40 \mathrm{TeV}$ and $\sim 4$ events at $\sqrt{s}=15 \mathrm{TeV}$. Work is in progress to develop cuts to reduce the gluon exchange background.

If either of the methods to detect the mixed decay modes $W W \rightarrow \ell \bar{\nu} \bar{q} q$ prove practicable, they can also be applied to the $W W$ continuum signal. Including $W^{+} W^{-}, W^{+} W^{+}$, and $W^{-} W^{-}$, the net $W W$ continuum signal with $|y w|<1.5$ and $M_{W W}>1 \mathrm{TeV}$ is $\sim 2 / 3$ of the $H \rightarrow W^{+} W^{-}$signal for $m_{H}=1 \mathrm{TeV}$.

Though we have pessimistically concentrated here on continuum signals of strong $W W$ scattering, where there are strong interactions there are probably also resonances. As a concrete example consider the techni-rho meson of $N_{c}=4$ technicolor, with a mass of 1.8 $\mathrm{TeV}$ and width of $260 \mathrm{GeV}$. In pp colliders both $\bar{q} q \rightarrow \rho_{T}^{35}$ and $W W \rightarrow \rho_{T}{ }^{2}$ contribute comparably to the production cross section. In analogy to the hadronic $\rho$ we expect the charged $\rho_{T}$ to decay predominantly to $W Z, \rho_{T}^{ \pm} \rightarrow W^{ \pm} Z$, which can be detected in the leptonic decay mode $W Z \rightarrow \ell \bar{\nu} \ell \bar{\ell}$ that occurs with a $1 \%$ branching ratio for $\ell=e$ or $\mu$. Then at the SSC with $10^{4} \mathrm{pb}^{-1}$ the signal in the central region, $\left|y_{w}\right|$ and $\left|y_{z}\right|<1.5$, is 12 events over a $\bar{q} q \rightarrow W Z$ background of only 1 event, while the LHC signal is 1 event over a somewhat smaller background. ${ }^{2}$ 


\subsection{Prospects for the ELOISATRON}

I have argued in Section 4.2 that the SSC parameters, $\sqrt{s}=40 \mathrm{TeV}$ and $\mathcal{L}=10^{33} \mathrm{~cm}^{-2} \mathrm{~s}^{-1}$, provide an assured capability to see the signals of a strongly interacting symmetry breaking sector above $1 \mathrm{TeV}$. The SSC occupies strategic ground in that it is close to being the minimal machine about which this statement can be made. We have seen in Section 4.2 that the numbers of events are only a few ten's per leptonic channel and a few hundred's for the mixed lepton-hadron channel if the latter proves to be detectable. If such signals are observed there will be an irresistable motivation to find a way to a higher energy facility where the new quanta could be produced in sufficient numbers to begin detailed studies. This higher energy facility could be the $200 \mathrm{TeV}$ pp collider that is the focus of this workshop or it might be an $e^{+} e^{-}$collider. (In the latter case the center of mass energy would clearly need to be much greater than the $2 \mathrm{TeV}$ being considered for CLIC.) Here I consider only the pp collider option.

The technological, financial, social, and political implications of such a device are enormous and to me at least they are essentially imponderable. It is however straightforward to estimate the physics signals at such a collider if the $S U(2)_{L} \times U(1)_{Y}$ breaking condensate arises from a strong fifth force with associated quanta above $1 \mathrm{TeV}$. To begin we can simply apply the models considered in Section 4.2 to the ELOISATRON energy. The result is table 3 , where we see that the signals of the $1 \mathrm{TeV}$ Higgs boson and the strong scattering model are an order of magnitude larger than at ihe SSC and two orders larger than at the LHC. The effective luminosity that accounts for both signal and background was given in eq. (4.5). All three tables assume the same integrated luminosity, $10^{4} \mathrm{pb}^{-1}$. Like the photon-photon scattering cross section, these $W W$ fusion cross sections grow with energy. We are not therefore faced with the same imperative to raise the luminosity that is implied by annihilation and hard scattering subprocess cross sections that scale like $1 / \hat{s}$ (though the maximum useable luminosity would obviously be desireable).

Table 4 underestimates the yield of quanta from a strong $\mathcal{L}_{S B}$ by at least one order of magnitude and perhaps more. For the signals of table 4 we are using the ELOISATRON as a source of colliding longitudinally polarized $W$ beams, the precise analogue of a pionpion scattering facility in hadron physics. The strong scattering model extrapolates the low energy theorems, eqs. $(2.16-2.20)$, as in eq. (3.16) (see reference (2) for the formulation in the other partial waves). It therefore represents only the three partial waves that dominate at threshold, $I, J=:(0,0),(1,1),(2,0)$. At ELOISATRON energies many partial waves contribute, up to $\ell=p R$, as in hadron scattering where here $R$ is the $W_{L}$ radius. A geometrical cross section may be a more appropriate estimate,

$$
\dot{\sigma}\left(W_{L} W_{L}\right)_{>1} \mathrm{TeV} \cong \pi R^{2}
$$

where $R$ is the $W_{L}$ radius. For comparison the strong scattering model gives

$$
\hat{\sigma}\left(W_{L} W_{L}\right)_{1-2} \mathrm{TeV} \cong \frac{1}{2 v^{2}} \cong O(\ln b) .
$$


To compare eqs. (4.11) and (4.12) we need an estimate for the radius $R$. Hadron radii might be characterized by their "pion clouds" extending to $R \cong m_{\pi}^{-1}$ which in the present context becomes $R \cong M_{W}^{-1}$. Then eq. (4.11) gives $\sim 100 \mathrm{nb}$, two order of magnitude bigger than eq. (4.12). A more conservative estimate in a technicolor model would use the confinement radius, $R \cong \Lambda_{T C}^{-1} \cong v^{-1}$, in which case eq. (4.11) is $\pi / v^{2} \cong O(10) \mathrm{nb}$. Table 4 is probably conservative by at least one order of magnitude. At ELOISATRON energies inelasticity will begin to set in and $n>2$ particle final states will begin to emerge.

If the new quanta carry QCD color or have colored constituents then they can be directly produced by gluon-gluon fusion. In that case we would expect still larger cross sections. 


\section{CONCLUSION}

There is always an element of iisk that accompanies investment in a major new research facility. Even if the instrument performs as designed, the fact that ve are exploring unknown phenomena means we cannot predict whether the discoveries will justify the investment. The historical record is reassuring: scientifically our investments up to now have proved well founded, and from scientific succsss other benefits are sure to come, both indirect and direct. Nevertheless as the scale of investment grows, the risk also increases. Though it will always continue to be true that some of the most important discoveries are completely unanticipated, it also becomes important to know in advance of phimoneisa that the new facility is sure to elucidate.

Such concerns apply to the LHC and SSC projected for the 1990's and would appiy with even greater force to the ELOISATRON in the next century. Two pnints from these: lectures are relevant to these concerns:

- If the signals of a strongly interacting symmetry breaking sector above $1 \mathrm{TeV}$ are found during the 1990's, the physics case for a facility with the capability of the ELOISATRON will be as clear as was the case for the particle accelerators built in the 50's and 60's to study the emerging physics of hadrons. The overriding concern of particle physics will then be to solve the technical, economic and political problems that will allow us to study in detail the new TeV-scale force and particles. Without such detailed studies high energy physics will be at an impasse. Therretical efforts to skip directly to the Planck scale will prove to be as premature as was the effort to unify gravity and electromagnetism without incorporating the weak and strong nuclear forces. And nonaccelerator experimental studies, while valuable as exploxatory probes, can never replace the controlled accelerator laboratory setting for the detailed studies that would be needed.

- If the $S U(2)_{L} \times U(1)_{Y}$ symmetry is broken by a new strong force and quanta above 1 $\mathrm{TeV}$, the only likely prospect to discover that new force in the 1990's lies with the SSC. The SSC is sure to establish if the force is weak or strong, to determine if the new quanta are lighter or heavier than $1 \mathrm{TeV}$, and probably to discover the new quarita provided the lightest of them are not much heavier than the $O(2) \mathrm{TeV}$ scale of the unitarity limit, eq. (3.2). Only by measuring the symmetry breaking force directly in $W_{L} W_{L}$ scattering between 1 and 2 TeV will we know for sure if it is strong. Other predictions, such as the pseudeGoldstone bosons of some technicolor theories, are model dependent and would not have unambiguous interpretations if found. Even with the luminosity upgrades that have been ccrsiderec, a $15 \mathrm{TeV}$ pp collider has little prospect to detect the 1-2 TeV $W_{L} W_{L}$ strong scattering signal. Though capable of important discoveries it cannot replace the SSC in this respect. 
In this sense the SSC and the ELOISATRON are strategically linked. If a strongly coupled symmetry breaking sector exists above $1 \mathrm{TeV}$, we will need to build a facility with the physics reach of the ELOISATRON to study its properties. But only by constructing the SSC are we likely to establish in this century whether a strongly coupled symmetry breaking sector exists in nature.

\section{DISCLAIMER}

This report was pirepared as in account of work sponsored by an agency of the United States Government. Neither the United States Gnvernment nor any agency thereof, nor any of their employees, makes any warranty, express or implied, or assumes any legal liability or responsibility for the accuracy, completeness, or usefulness of any information, apparatus, product, or process disclosed, or represents that its use would not infringe privalely owned rights. Refer. ence herein to any specific commercial product, process, or service by trade name, trademark, manufacturer, or otherwise does not necessarily constitute or imply its endorsement, recommendation, or favoring by the United States Government or any agency thereof. The views and opinions of authors expressed herein do not necessarily state or reflect those of the United States Government or any agency thereof. 


\section{REFERENCES}

1. S.L. Glashow, Nucl. Phys. 22, 5 (1961); S. Weinberg, Phys. Rev. Lett. 19, 1264 (1967); A. Salam, in Proc. Sth Nobel Symp., ed. N. Svartholm (Almqvist and Wiksells, Stockholm, 1968) p. 367.

2. M.S. Chanowitz and M.K. Gaillard, Nucl. Phys. B261, 379 (1985).

3. M.S. Chanowitz, M. Golden, H. Georgi, Phys. Rev. Lett. 57, 2344 (1986); Phys. Rev. D36, 1490 (1987).

4. S. Weinberg, Phys. Rev. D13, 974 (1976); D20: 1277 (1979); L. Susskind, Phys. Rev. D20, 2619 (1979).

5. R.N. Cahn and S. Dawson, Phys. Lett. 136B, 196 (1984); E 138B: 464 (1984).

6. M.S. Chanowitz and M.K. Gaillard, Phys. Lett. 142B, 85 (1984); S. Dawson, Nucl. Phys. B29, 42 (1985); G. Kane, W. Repko, W. Rolnick, Phys. Lett. 148B, 367 (1984).

7. The Feasibility of Experiments at High Luminosity at the LHC, ed. J. Mulvey, CERN 8\&.02 (1988).

8. M. Chanowitz and R. Cahn, Phys. Rev. Lett. 56, 1327 (1986).

9. B. Cox and F. Gilman, p. 87, Proc. 1984 Summer Study on Design and Utilization of the SSC, eds. R. Donaldson and J. Morfin, American Physical Society.

10. M. Bento and C.H. Llewellyn Smith, Nucl. Phys. B289, 36 (1987); G. Altarelli, B. Mele, F. Pitolli, Nucl. Phys. B287, 205 (1987); J. Gunion, A. Tofighi-Niaki, Phys. Rev. D36, 2671 (1987).

10a. M. Chanowitz, Ann. Rev. Nucl. Part. Sci. 38, 323 (1988).

11. M. Cornwall, D. Levin, G. Tiktopoulos, Phys. Rev. D10, 1145 (1974).

12. G. Gounaris, R. Kogerler, H. Neufeld, Phys. Rev. D34, 3257 (1986).

13. C. Vayonakis, Lett. Nuovo Cim. 17, 383 (1976).

14. B.W. Lee, C. Quigg, H. Thacker, Phys. Rev. D16, 1519 (1977).

15. M. Chanowitz, M. Furman, I. Hinchliffe, Phys. Rev. Lett. 78B, 285 (1978); Nucl. Phys. B153, 402 (i979).

16. M. Duncan, G. Kane, W. Repko, Nucl. Phys. B272, 571 (1986).

17. D. Dicus, R. Vega, Phys. Rev. Lett. 57, 1110 (1986).

18. J. Gunion, J. Kalinowski, A. Tofighi-Niakis, Phys. Rev. Lett. 57, 2351 (1986).

19. R.N. Cahn et al., Phys. Rev. D35, 1626 (1987).

20. J. Donoghue, C. Ramirez, G. Valencia, Phys. Rev. D38, 2195 (1988).

21. See. ref. (16).

22. G. Altarelli et al., ref. (10).

23. R. Cahn, Nucl. Phys. B255, 341 (1985).

24. See ref. (18).

25. H. Georgi et al., Phys. Rev. Lett. 40, 692 (1978). 
26. D. Froidevaux et al., p. 61, Proc. Workshop on Physics at Future Accelerators, ed. J. Mulvey, CERN 87-07 (1987).

27. R. Cahn et al., p. 20, Proc. Workshop on Experiments, Detectors, and Experimental Areas for the SSC, July 7-17, 1987, Berkeley, eds. R. Donaldson and M. Gilchriese (World Scientific, Singapore, 1988).

28. W. Stirling et al., Phys. Lett. 163B, 261 (1985); J. Gunion et al., Phys. Lett. 163B, 389 (1985).

29. J. Gunion and M. Soldate, Phys. Rev. D34, 826 (1986).

30. A. Savoy-Navarro, p. 68 , ref. (27).

31. R. Kleiss, W. Stirling, Phys. Lett. 200B, 193 (1988); W.J. Stirling, these proceedings.

32. G. Herten, p. 103, ref. (27).

33. M. Chanowitz, M. Golden, Phys. Rev. Lett. 61, 1053 (1988). Due to a programming error the gluon exchange background was underestimated in this paper-see ref. (34) and Chanowitz and Golden, in preparation.

34. D. Dicus and R. Vega, U.C. Davis preprint (1988).

35. E. Eichten et al., Rev. Mod. Phys. 56, 579 (1984). 
Table 1: Effective $W_{L}^{+} W_{L}^{-}$luminosity as a function of $\sqrt{s_{q q}} / \sqrt{s_{W W}}$.

\begin{tabular}{|c|c|c|c|c|c|c|}
\hline$\frac{1}{\sqrt{x}}=\sqrt{\frac{s_{q q}}{s W W}}$ & 1 & 2 & 3 & 4 & 5 & 10 \\
\hline$\frac{16 \pi}{\alpha_{W}^{2}} \frac{\partial \mathcal{L}}{\partial x}$ & 0 & 0.9 & 6 & 17 & 36 & 270 \\
\hline
\end{tabular}

Table 2: Yields at a $16 \mathrm{TeV}$ pp collider from $W W$ fusion and $\bar{q} q$ annihilation in events per $10^{4} \mathrm{pb}^{-1}$. Cuts imposed are $\left|y_{w}\right|<1.5$ and $s_{w w}>1 \mathrm{TeV}^{2}$. No gauge boson decay branching ratios are included.

\begin{tabular}{|l|c|c|c|}
\hline$\sqrt{s}=16 \mathrm{TeV}$ & $1 \mathrm{TeV}$ Higgs & $\begin{array}{c}\text { Strong Scattering } \\
\text { Model }\end{array}$ & $\bar{q} q$ annihilation \\
\hline$Z Z$ & 110 & 50 & 100 \\
\hline$W^{+} W^{-}$ & 220 & 60 & 440 \\
\hline$W^{+} Z+W^{-} Z$ & & 60 & 180 \\
\hline$W^{+} W^{+}+W^{-} W^{-}$ & & 70 & \\
\hline
\end{tabular}

Table 3: As in Table 2, for a $40 \mathrm{TeV}$ pp collider.

\begin{tabular}{|l|c|c|c|}
\hline$\sqrt{s}=40 \mathrm{TeV}$ & $1 \mathrm{TeV}$ Higgs & $\begin{array}{c}\text { Strong Scattering } \\
\text { Model }\end{array}$ & $\bar{q} q$ annihilation \\
\hline$Z Z$ & 890 & 490 & 370 \\
\hline$W^{+} W^{-}$ & 1800 & 630 & 1600 \\
\hline$W^{+} Z+W^{-} Z$ & & 670 & 580 \\
\hline$W^{+} W^{+}+W^{-} W^{-}$ & & 710 & \\
\hline
\end{tabular}

Table 4: As in Table 2, for a $200 \mathrm{TeV}$ pp collider.

\begin{tabular}{|l|c|c|c|}
\hline$\sqrt{s}=200 \mathrm{TeV}$ & $1 \mathrm{TeV}$ Higgs & $\begin{array}{c}\text { Strong Scattering } \\
\text { Model }\end{array}$ & $\bar{q} q$ annihilation \\
\hline$Z Z$ & 9800 & 6100 & 1800 \\
\hline$W^{+} W^{-}$ & 20000 & 8000 & 8000 \\
\hline$W^{+} Z+W^{-} Z$ & & 9500 & 2500 \\
\hline$W^{+} W^{+}+W^{-} W^{-}$ & & 8900 & \\
\hline
\end{tabular}




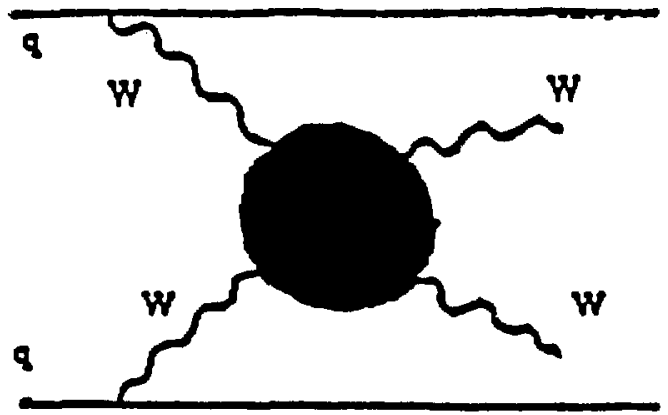

Figure 1: Production of $W W$ pairs by $W W$ fusion. 


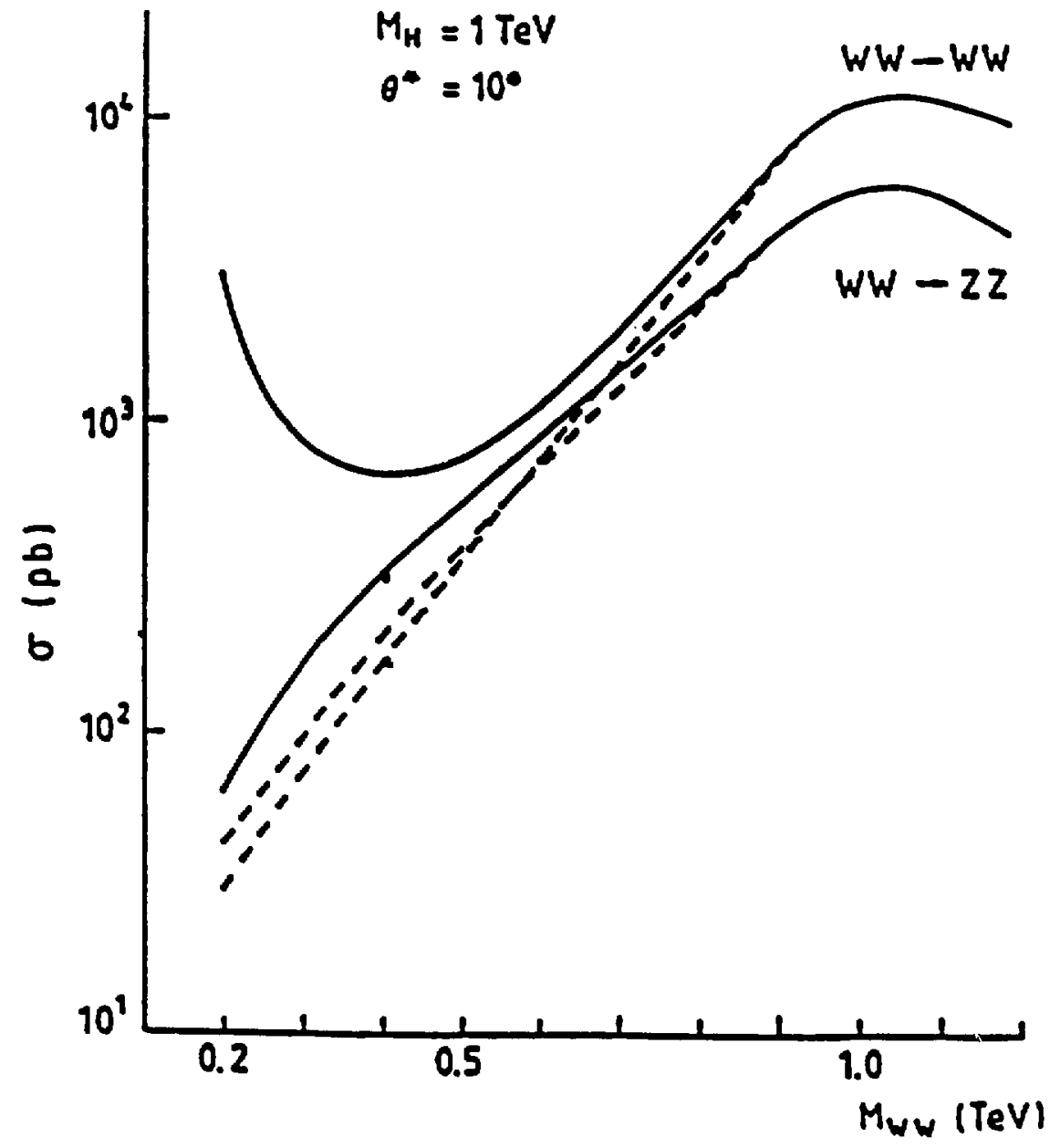

Figure 2: Comparison of $W W$ cross sections in unitary gauge and with use of the equivalence theorem (from Bento and Llewellyn Smith, ref. (10)). 

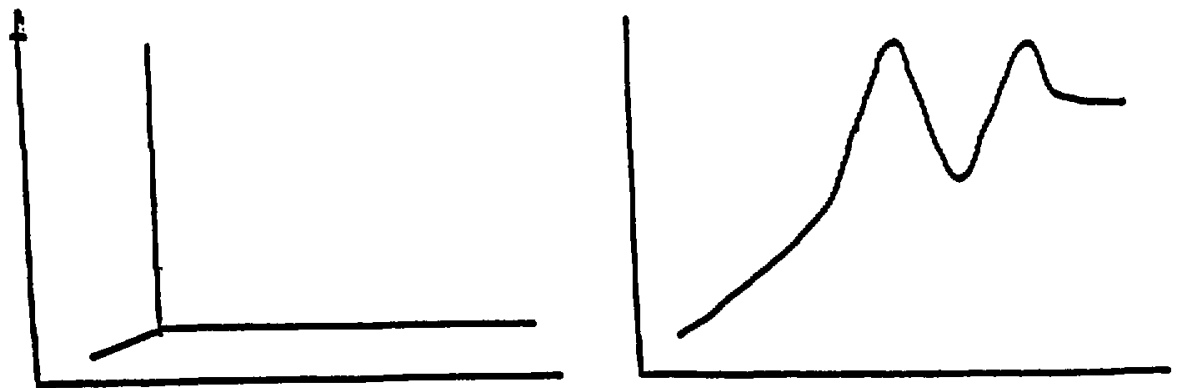

Figure 3: Typical behavior of partial wave amplitudes for $W_{L} W_{L}$ scattering. The plot on the left represents a weak coupling model with a narrow (Higgs) resonance below $1 \mathrm{TeV}$. The plot on the right shows strong coupling behavior, with saturation of unitarity and broad resonances in the 1-2 $\mathrm{TeV}$ region. 

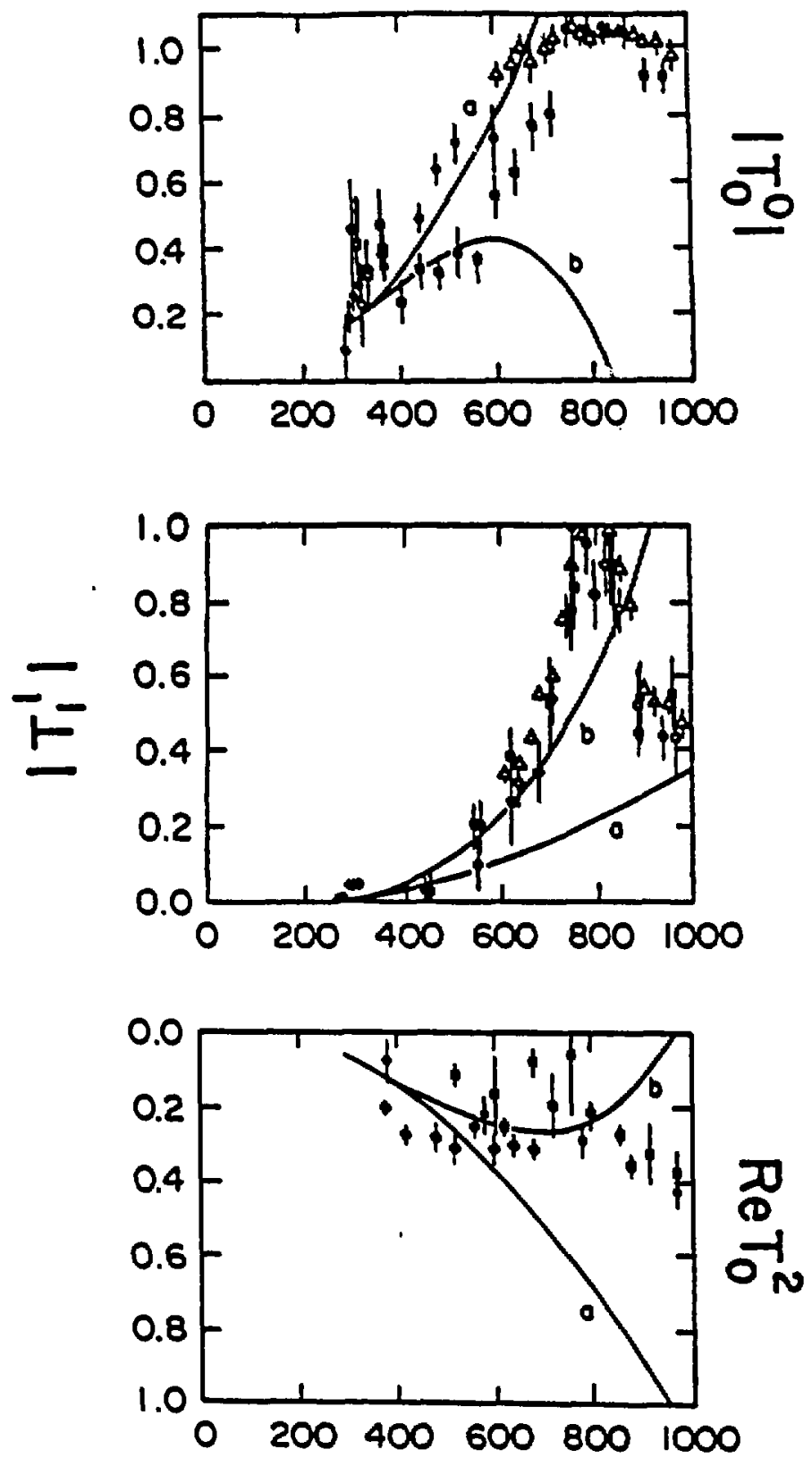

Figure 4: Data for $\pi \pi$ partial wave amplitudes compared with extrapolated low energy theorems (curves a) as in equation 3.12 (from ref. 20). 


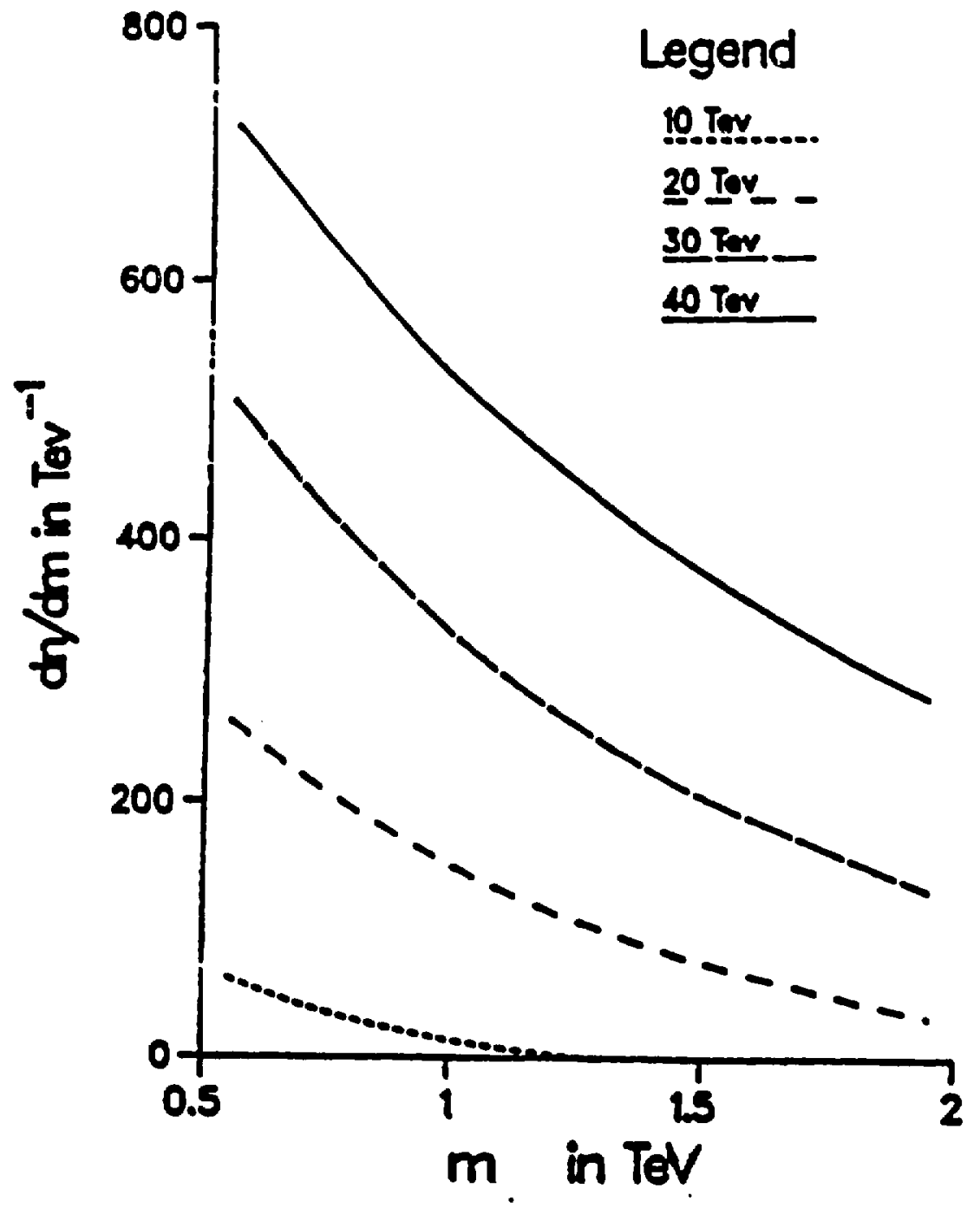

Figure 5: Differential yield per $10 \mathrm{fb}^{-1}$ with respect to $m_{z z}$ for the model of equation 3.12, at pp colliders of $10,20,30$, and $40 \mathrm{TeV}$ (from ref. (2)). Rapidity $|y z|<1.5$ is required. 


$$
Z Z: m_{H}=1 \text { Tev }
$$

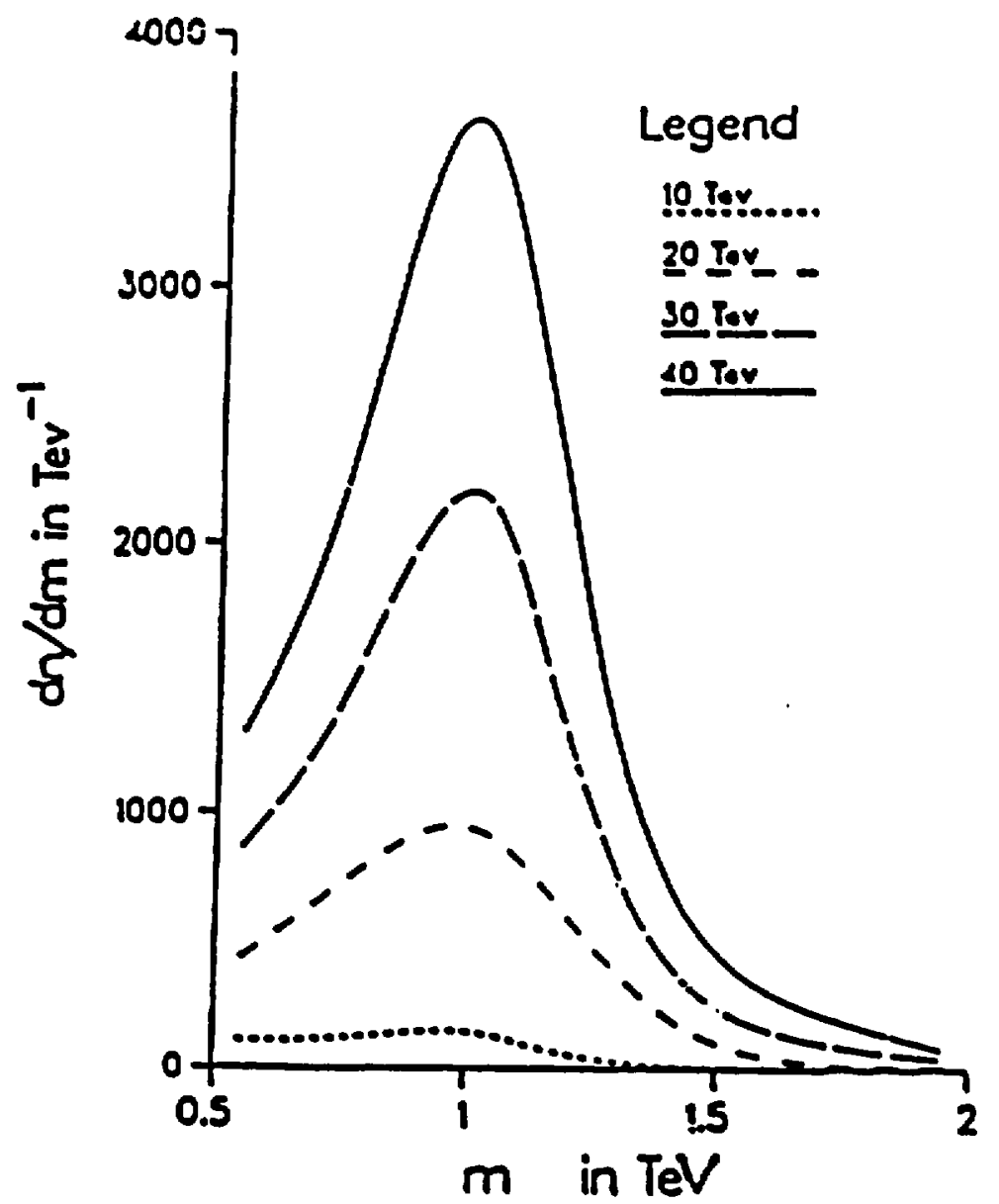

Figure 6: Differential yield per $10 \mathrm{fb}^{-1}$ with respect to $m_{z Z}$ for production of a $1 \mathrm{TeV}$ Higgs boeon at pp colliders of 10, 20,30, and $40 \mathrm{TeV}$ (from ref. (2)). Rapidity $\left|y_{2}\right|<1.5$ is required. 


\section{Z Z : signals + background $40 \mathrm{Tev}$}

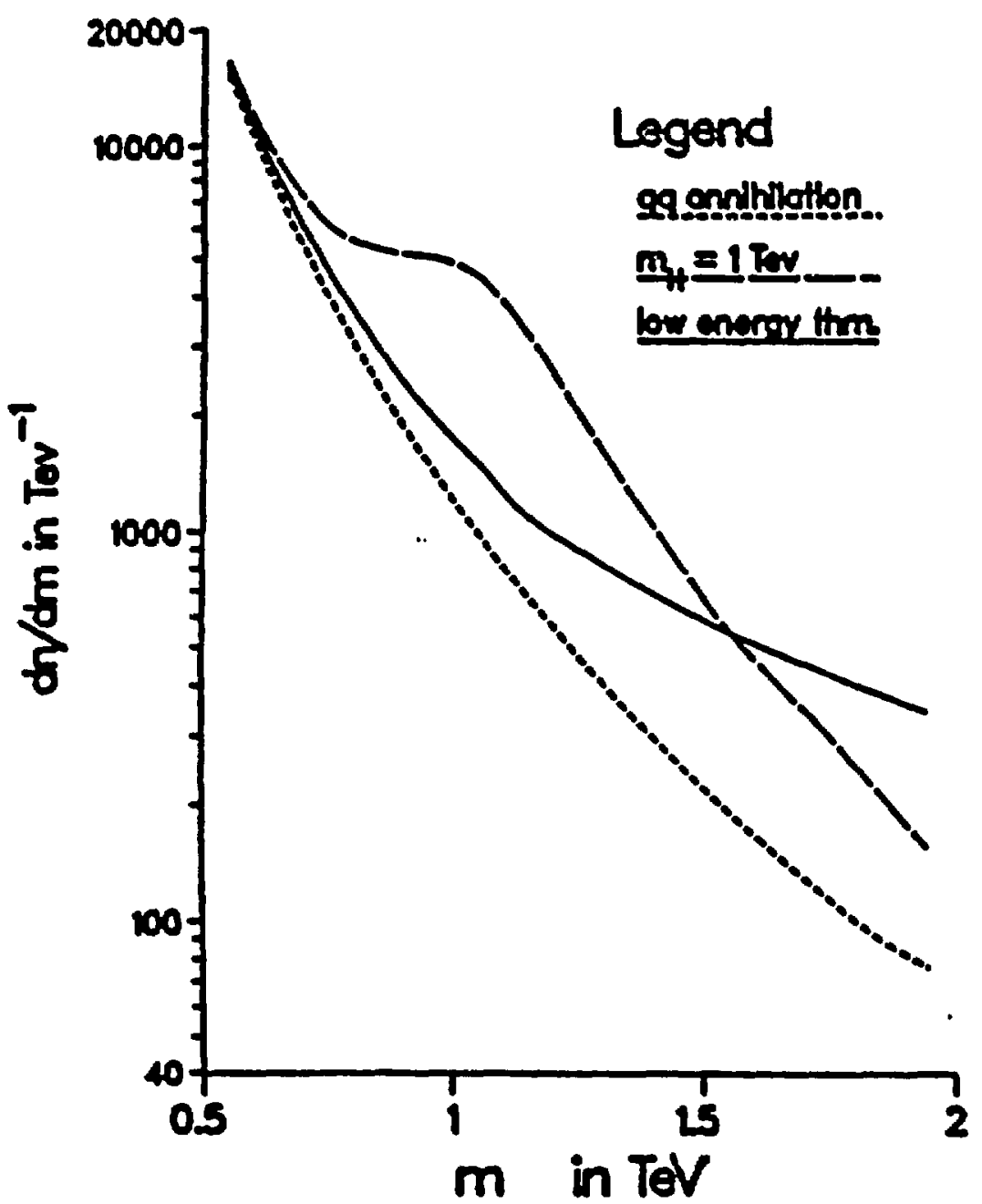

Figure 7: Differential yield per $10 \mathrm{fb}^{-1}$ with respect to $m_{z z}$ of the $1 \mathrm{TeV}$ Higgs boos and the model of equation 3.12, both shown as increments on the $\bar{q} q$ annihilation background with $\sqrt{s}=40 \mathrm{TeV}$ (from ref. (2)). Rapidity $\left|y_{z}\right|<1.5$ is required. 